\title{
Quantum-proof randomness extractors via operator space theory
}

\author{
Mario Berta, ${ }^{1, *}$ Omar Fawzi, $, 2,3,4$ and Volkher B. Scholz ${ }^{4} \ddagger$ \\ ${ }^{1}$ Institute for Quantum Information and Matter, Caltech, Pasadena, CA 91125, USA \\ ${ }^{2}$ Department of Computing and Mathematical Sciences, Caltech, Pasadena, CA 91125, USA \\ ${ }^{3}$ ENS de Lyon, UMR 5668 LIP - CNRS - UCBL - INRIA - Université de Lyon, 69364 Lyon, France \\ ${ }^{4}$ Institute for Theoretical Physics, ETH Zurich, 8093 Zürich, Switzerland
}

\begin{abstract}
Quantum-proof randomness extractors are an important building block for classical and quantum cryptography as well as for device independent randomness amplification and expansion. It is known that some constructions are quantum-proof whereas others are provably not [Gavinsky et al., STOC'07]. We argue that the theory of operator spaces offers a natural framework for studying to what extent extractors are secure against quantum adversaries: we first phrase the definition of extractors as a bounded norm condition between normed spaces, and then show that the presence of quantum adversaries corresponds to a completely bounded norm condition between operator spaces. From this we show that very high min-entropy extractors as well as extractors with small output are always (approximately) quantum-proof.

We also study a generalization of extractors called randomness condensers. We phrase the definition of condensers as a bounded norm condition and the definition of quantum-proof condensers as a completely bounded norm condition. Seeing condensers as bipartite graphs, we then find that the bounded norm condition corresponds to an instance of a well studied combinatorial problem, called bipartite densest subgraph. Furthermore, using the characterization in terms of operator spaces, we can associate to any condenser a Bell inequality (two-player game) such that classical and quantum strategies are in one-to-one correspondence with classical and quantum attacks on the condenser. Hence, we get for every quantum-proof condenser (which includes in particular quantum-proof extractors) a Bell inequality that can not be violated by quantum mechanics.
\end{abstract}

\section{INTRODUCTION}

In cryptographic protocols such as in key distribution and randomness expansion, it is often possible to guarantee that an adversary's knowledge about the secret $N$ held by honest players is bounded. The relevant quantity in many settings is the adversary's guessing probability of the secret $N$ given all his knowledge. However, the objective is usually not to create a secret that is only partly private but rather to create a (possibly smaller) secret that is almost perfectly private. The process of transforming a partly private string $N$ into one that is almost uniformly random $M$ from the adversary's point of view is called privacy amplification $[2,3]$. In order to perform privacy amplification, we apply to $N$ a function chosen at random from a set of functions $\left\{f_{s}\right\}$ that has the property of being a randomness extractor. Randomness extractors are by now a standard tool used in many classical and quantum protocols. They are for example an essential ingredient in quantum key distribution and device independent randomness expansion protocols [33, 43]. For such applications, it has been only relatively recently realized [33] that it is crucial to explicitly consider quantum adversaries. It is by no means obvious that a quantum adversary also satisfying the guessing probability constraint on $N$ would not be able to have additional knowledge about the output $M$. In fact, as explained below, we know of an extractor construction that becomes useless against quantum adversaries [16].

We believe that in the same way as communication complexity and Bell inequalities (multi prover games), the setting of randomness extractors provides a beautiful framework for studying the power and limitations of a quantum memory compared to a classical one. Here we argue that the theory of operator spaces, sometimes also called "quantized functional analysis", provides a natural arena for studying this question. This theory has already been successfully applied in the context of understanding Bell inequality violations, see $[18,19]$ and references therein.

This document is structured as follows. In the next two subsections we define (quantum-proof) randomness extractors (Section I A) and condensers (Section IB), and give a summary of the known results that are relevant to our discussion. In Section II we present an overview of our results (leaving out all the proofs). This is then followed by some open questions stated in Section III. For the main body of the paper, we start with basic preliminaries on the theory of normed spaces and operator spaces (Section IV). In Section V we prove our results about quantum-proof extractors. The last section is devoted to the proofs of our results concerning condensers (Section VI). Some technical arguments are deferred to the Appendices (Appendix A-B).

\footnotetext{
* berta@caltech.edu

$\dagger$ omar.fawzi@ens-lyon.fr

$\ddagger$ scholz@phys.ethz.ch
} 


\section{A. Randomness extractors}

Extractors map a weakly random system into (almost) uniform random bits, with the help of perfectly random bits called the seed. We use $N=2^{n}$ to denote the input system (consisting of strings of $n$ bits), $M=2^{m}$ (bit-strings of length $m$ ) to denote the output system, and $D=2^{d}(d$ bits) to denote the seed system. Note that in a slight abuse of notation, we use the same letters for the actual system as well as its dimension as a linear vector space. An extractor is then a family of functions $\left\{f_{1}, \ldots, f_{D}\right\}$ with $f_{s}: N \rightarrow M$ satisfying the following property. For any random variable on $N$ with

$$
H_{\min }(N):=-\log p_{\text {guess }}(N) \geq k,
$$

where $p_{\text {guess }}(N)$ denotes the maximal probability of guessing the input, and an independent and uniform seed $U$, the random variable $f_{U}(N)$ has a distribution which is $\varepsilon$-close in total variation distance to the uniform distribution $v_{M}$ on $M$. For us, it is more convenient to state the definition in terms of probability distributions. For this we associate to the functions $f_{s}$ an $M \times N$ matrix $F_{s}$ where the entry $(y, x)$ is equal to one if $f_{s}(x)=y$ and zero otherwise. With this notation, we have for any probability distribution $P_{N}$ of a random variable on $N, F_{s}\left(P_{N}\right)$ is the distribution of $f_{s}(N)$. That is, a $(k, \varepsilon)$-extractor satisfies the following property. For all input probability distributions $P_{N}$ with $H_{\min }(N)_{P} \geq k$,

$$
\left\|\frac{1}{D} \cdot \sum_{s=1}^{D} F_{s}\left(P_{N}\right)-v_{M}\right\|_{\ell_{1}} \leq \varepsilon .
$$

This definition is also referred to as weak extractors. An important special case of extractors are strong extractors [27], for which the seed is part of the output, i.e., the output space has the form $M=D \times M^{\prime}$ and $f_{s}(x)=\left(s, f_{s}^{\prime}(x)\right)$ for some function $f_{s}^{\prime}: N \rightarrow M^{\prime}$ (with $M^{\prime}=2^{m^{\prime}}$ ). This means that the invested randomness, the seed $D$, is not used up and can safely be reused later. The condition (2) then reads as

$$
\frac{1}{D} \cdot \sum_{s=1}^{D}\left\|F_{s}^{\prime}\left(P_{N}\right)-v_{M^{\prime}}\right\|_{\ell_{1}} \leq \varepsilon
$$

Such objects are needed for privacy amplification, since the eavesdropper is allowed to know which function is applied.

We now briefly discuss the parameters for which strong extractors exists. Typically we want to maximize the output length $m$ and minimize the seed length $d$. Radhakrishnan and Ta-Shma [30] show that every strong $(k, \varepsilon)$-extractor necessarily has

$$
m \leq k-2 \log (1 / \varepsilon)+O(1) \quad \text { and } \quad d \geq \log (n-k)+2 \log (1 / \varepsilon)-O(1) .
$$

Using the probabilistic method one can show that random functions achieves these bounds up to constants [30, 36]. There exists a strong $(k, \varepsilon)$-extractor with

$$
m=k-2 \log (1 / \varepsilon)-O(1) \quad \text { and } \quad d=\log (n-k)+2 \log (1 / \varepsilon)+O(1) .
$$

Probabilistic constructions are interesting, but for applications we usually need explicit extractors. Starting with the work by Nisan and Ta-Shma [26] and followed by Trevisan's breakthrough result [41] there has been a lot of progress in this direction, and there are now many constructions that almost achieve the converse bounds in (4) (see the review articles [35, 42]).

For applications in classical and quantum cryptography (see, e.g., [24, 33]) and for constructing device independent randomness amplification and expansion schemes (see, e.g., [8, 10, 11, 25]) it is important to find out if extractor constructions also work when the input source is correlated to another (possibly quantum) system $Q$. That is, we would like that for all classical-quantum input density matrices $\rho_{Q N}$ with conditional min-entropy

$$
H_{\min }(N \mid Q)_{\rho}:=-\log p_{\text {guess }}(N \mid Q)_{\rho} \geq k
$$

where $p_{\text {guess }}(N \mid Q)$ denotes the maximal probability of guessing $N$ given $Q$, the output is uniform and independent of $Q{ }^{1}$

$$
\left\|\frac{1}{D} \sum_{s=1}^{D}\left(\operatorname{id}_{Q} \otimes F_{s}\right)\left(\rho_{Q N}\right)-\rho_{Q} \otimes v_{M}\right\|_{1} \leq \varepsilon
$$

\footnotetext{
${ }^{1}$ Other notions for weaker quantum adversaires have also been discussed in the literature, e.g., in the bounded storage model (see [12, Section 1] for a detailed overview).
} 
and $\|\cdot\|_{1}$ denotes the trace norm (the quantum extension of the $\ell_{1}$-norm). Similarly, the corresponding condition for quantum-proof strong extractors reads

$$
\frac{1}{D} \sum_{s=1}^{D}\left\|\left(\operatorname{id}_{Q} \otimes F_{s}^{\prime}\right)\left(\rho_{Q N}\right)-\rho_{Q} \otimes v_{M^{\prime}}\right\|_{1} \leq \varepsilon .
$$

König and Terhal [23, Proposition 1] observed that if we restrict the system $Q$ to be classical with respect to some basis $\{|e\rangle\}_{e \in Q}$ then every $(k, \varepsilon)$-extractor as in $(2)$ is also a $(k+\log (1 / \varepsilon), 2 \varepsilon)$-extractor in the sense of $(7)$ (and the analogue statement for strong extractors is a special case of this). That is, even when the input source is correlated to a classical system $Q$, every extractor construction still works (nearly) equally well for extracting randomness. However, if $Q$ is quantum no such generic reduction is known and extractor constructions that also work for quantum $Q$ are called quantum-proof. ${ }^{2}$ Examples of (approximately) quantum-proof extractors include:

- Spectral $(k, \varepsilon)$-extractors are quantum-proof $(k, 2 \sqrt{\varepsilon})$-extractors [4, Theorem 4]. This includes in particular two-universal hashing [33, 40], two-wise independent permutations [37], as well as sample and hash based constructions [22].

- One bit output strong $(k, \varepsilon)$-extractors are quantum-proof strong $(k+\log (1 / \varepsilon), 3 \sqrt{\varepsilon})$-extractors [23, Theorem 1].

- Strong $(k, \varepsilon)$-extractors constructed along Trevisan's ideas [41] are quantum-proof $\operatorname{strong}(k+\log (1 / \varepsilon), 3 \sqrt{\varepsilon})$ extractors [12, Theorem 4.6] (see also [1]).

We emphasize that all these stability results are specifically tailored proofs that make use of the structure of the particular extractor constructions. In contrast to these findings it was shown by Gavinsky et al. [16, Theorem 1] that there exists a valid (though contrived) strong extractor for which the decrease in the quality of the output randomness has to be at least $\varepsilon \mapsto \Omega\left(m^{\prime} \varepsilon\right) .{ }^{3}$ As put forward by Ta-Shma [38, Slide 84], this then raises the question if the separation found by Gavinsky et al. is maximal, that is:

Is every $(k, \varepsilon)$-extractor a quantum-proof $(O(k+\log (1 / \varepsilon)), O(m \sqrt{\varepsilon}))$-extractor or does there exists an extractor that is not quantum-proof with a large separation, say $\varepsilon \mapsto\left(2^{m} \varepsilon\right)^{\Omega(1)}$ ?

We note that such a stability result would make every extractor with reasonable parameters (approximately) quantumproof.

\section{B. Randomness condensers}

In the general theory of pseudorandomness one interesting generalization of extractors are condensers. These objects were first defined in $[31,32]$ as an intermediate step in order to build extractors. For condensers the output is not necessarily close to the uniform distribution (as it is the case for extractors), but only close to a distribution with high min-entropy $k^{\prime}$. More precisely, a (weak) $\left(k \rightarrow_{\varepsilon} k^{\prime}\right)$-condenser is family of functions $\left\{f_{1}, \ldots, f_{D}\right\}$ with $f_{s}: N \rightarrow M$ such that for all input probability distributions $P_{N}$ with $H_{\min }(N)_{P} \geq k$

$$
H_{\min }^{\varepsilon}(M)_{\frac{1}{D} \sum_{s=1}^{D} F_{s}(P) \geq k^{\prime}}
$$

with the smooth min-entropy

$$
H_{\min }^{\varepsilon}(N)_{P}:=\sup _{R} H_{\min }(N)_{R}
$$

and the supremum over all probability distributions $R_{N}$ such that $\left\|R_{N}-P_{N}\right\|_{\ell_{1}} \leq \varepsilon$. Observe that when $k^{\prime}=\log M$, this is exactly the condition for being a (weak) $(k, \varepsilon)$-extractor. The reason this definition is called condenser is because we want constructions with $M<N$ so that the entropy is condensed in a smaller probability space. For the

\footnotetext{
2 Note that the dimension of $Q$ is unbounded and that it is a priori unclear if there exist any extractor constructions that are quantumproof (even with arbitrarily worse parameters). Furthermore, and in contrast to some claims in the literature, we believe that the question to what extent extractors are quantum-proof is already interesting for weak extractors. In particular, if weak extractors were perfectly quantum-proof then strong extractors would be perfectly quantum-proof as well (and we know that the latter is wrong [16]).

3 Since the quality of the output randomness of Gavinsky et al.'s construction is bad to start with, the decrease $\varepsilon \mapsto \Omega\left(m{ }^{\prime} \varepsilon\right)$ for quantum $Q$ already makes the extractor fail completely in this case.
} 
special case of strong condensers the output space has the form $M=D \times M^{\prime}$ and $f_{s}(x)=\left(s, f_{s}^{\prime}(x)\right)$ for some function $f_{s}^{\prime}: N \rightarrow M^{\prime}$, and the condition (9) then reads

$$
H_{\min }^{\varepsilon}\left(M^{\prime} D\right)_{\frac{1}{D} \sum_{s=1}^{D} F_{s}^{\prime}(P) \otimes|s\rangle\langle s|} \geq k^{\prime} .
$$

A (weak) quantum-proof condenser is as follows. For all classical-quantum input density matrices $\rho_{Q N}$ with conditional min-entropy $H_{\min }(N \mid Q)_{\rho} \geq k$, the output should be close to a distribution with high conditional min-entropy $k^{\prime}$

$$
H_{\min }^{\varepsilon}(M \mid Q)_{\frac{1}{D} \sum_{s=1}^{D}\left(\operatorname{id} \otimes F_{s}\right)(\rho)} \geq k^{\prime}
$$

with the smooth conditional min-entropy

$$
H_{\min }^{\varepsilon}(N \mid Q)_{\rho}:=\sup _{\sigma} H_{\min }(N \mid Q)_{\sigma}
$$

and the supremum over all classical-quantum density matrices $\sigma_{Q N}$ such that $\left\|\sigma_{Q N}-\rho_{Q N}\right\|_{1} \leq \varepsilon$. Similarly, the corresponding condition for quantum-proof strong condensers reads

$$
H_{\min }^{\varepsilon}\left(M^{\prime} D \mid Q\right)_{\frac{1}{D} \sum_{s=1}^{D}\left(\mathrm{id} \otimes F_{s}^{\prime}\right)(\rho) \otimes|s\rangle\langle s|} \geq k^{\prime} .
$$

We note that the works $[14,22,46]$ can be understood as results about quantum-proof condensers. One reason why we would like to understand to what extent condensers are quantum-proof is that the best known explicit extractor constructions are built from condensers (see the review article [42]).

As an extractor is a special case of a condenser with full output entropy $k^{\prime}=m$, one can understand the construction of Gavinsky et al. [16, Theorem 1] also as a valid randomness condenser that is not quantum-proof. But a condenser has the output entropy as an additional parameter, so it is natural to ask whether this construction is a quantum-proof condenser with slightly worse parameters, e.g., $k+c^{\prime \prime} \rightarrow_{c \varepsilon} k^{\prime}-c^{\prime}$ for some constants $c$, $c^{\prime}$ and $c^{\prime \prime}$. Note that when $c^{\prime}>0$, this does not correspond to an extractor condition anymore, as the output is only required to have large smooth min-entropy. It turns out that even if we relax the condition on the output min-entropy slightly, the condenser is still not quantum-proof. The reason is that the quantum attack given in [16] allows the adversary to correctly guess $\gamma$ bits of the output with a memory of size $O(\gamma \log n)$. Thus the smooth min-entropy of the output can be at most roughly $k^{\prime}-\gamma$.

\section{OVERVIEW OF RESULTS}

\section{A. Extractors}

A linear vector space which is equipped with a norm is called a normed space (we restrict ourselves to finitedimensional spaces). Special examples are the linear space $\mathbb{C}^{n}$, which can be equipped with the $\ell_{1}$-norm, the sum of all absolute values of vector entries, or the $\ell_{\infty}$-norm, the largest absolute value of vector entries. Both norms are useful for studying extractors, as the first norm encodes the normalization constraint (the inputs are probability distributions), while the second is just the exponential of the min-entropy. Linear maps between normed spaces are naturally equipped with norms, defined as the maximum norm of any output, given that the input had bounded norm. Of course, the norms on the input and the output spaces can be different. Our first result is that the extractor condition (2) can be rewritten as a condition on the norm of a linear map. In the expression (2), observe that

$$
P_{N} \mapsto \frac{1}{D} \sum_{s=1}^{D} F_{s}\left(P_{N}\right)
$$

is a linear map. In addition, as $P_{N}$ is a probability distribution, we can write $\nu_{M}=\left(\mathbf{1}^{T} P_{N}\right) \nu_{M}$, where $\mathbf{1}^{T}=$ $(1, \ldots, 1) \in \mathbb{R}^{N}$ is the vector with all ones. As a result,

$$
P_{N} \mapsto \frac{1}{D} \sum_{s=1}^{D} F_{s}\left(P_{N}\right)-\left(\mathbf{1}^{T} P_{N}\right) \nu_{M}
$$

is a linear function in the input distribution. We can associate to an extractor Ext $=\left\{f_{s}\right\}_{s \in D}$ a linear map from $N$ to $M$ given by

$$
\Delta[\operatorname{Ext}]\left(P_{N}\right):=\frac{1}{D} \sum_{s=1}^{D} F_{s}\left(P_{N}\right)-\left(\mathbf{1}^{T} P_{N}\right) \nu_{M}
$$


Using this notation, the extractor condition can be written as follows: for all distributions $P_{N}$ with $\left\|P_{N}\right\|_{\ell_{1}}=1$ and $H_{\min }(N)_{P} \geq k,\|\Delta[\operatorname{Ext}](P)\|_{\ell_{1}} \leq \varepsilon$. In order to capture the input constraints on $P_{N}$ we now define for $k \in(0, \infty)$ the $\cap\left\{2^{k} \ell_{\infty}, \ell_{1}\right\}$-norm as

$$
\left\|P_{N}\right\|_{\cap\left\{2^{k} \ell_{\infty}, \ell_{1}\right\}}:=\max \left\{2^{k}\left\|P_{N}\right\|_{\ell_{\infty}},\left\|P_{N}\right\|_{\ell_{1}}\right\}
$$

We then get the bounded norm

$$
\left\|\Delta[\mathrm{Ext}]: \cap\left\{2^{k} \ell_{\infty}, \ell_{1}\right\} \rightarrow \ell_{1}\right\| \equiv\|\Delta[\mathrm{Ext}]\|_{\cap\left\{2^{k} \ell_{\infty}, \ell_{1}\right\} \rightarrow \ell_{1}}:=\operatorname{uP}_{\left\|P_{N}\right\|_{\cap\left\{2^{k} \ell_{\infty}, \ell_{1}\right\}} \leq 1}\left\|\Delta[\operatorname{Ext}]\left(P_{N}\right)\right\|_{\ell_{1}},
$$

that gives the following alternative characterization for extractors.

Proposition II.1. Let Ext $=\left\{f_{s}\right\}_{s \in D}$ and $\Delta[\mathrm{Ext}]$ as defined in (17). Then, we have

$$
\begin{aligned}
\| \Delta[\text { Ext }]: \cap\left\{2^{k} \ell_{\infty}, \ell_{1}\right\} \rightarrow \ell_{1} \| \leq \varepsilon & \Rightarrow \quad \text { Ext is a }(k, \varepsilon) \text { - extractor } \\
\text { Ext is a }(k-1, \varepsilon) \text { - extractor } & \Rightarrow \quad \| \Delta[\text { Ext }]: \cap\left\{2^{k} \ell_{\infty}, \ell_{1}\right\} \rightarrow \ell_{1} \| \leq 3 \varepsilon .
\end{aligned}
$$

Note that strong extractors are covered by this as a special case. To make this explicit we associate to a strong extractor Ext $=\left\{f_{s}\right\}_{s \in D}$ the linear map

$$
\Delta[\mathrm{Ext}]_{S}\left(P_{N}\right):=\frac{1}{D} \sum_{s=1}^{D} F_{s}\left(P_{N}\right) \otimes|s\rangle\left\langle\left. s\right|_{D}-\left(\mathbf{1}^{T} P_{N}\right) \nu_{M^{\prime}} \otimes \nu_{D}\right.
$$

and the corresponding statement can then be read off by replacing $\Delta[$ Ext $]$ with $\Delta[\text { Ext }]_{S}$ in Proposition II.1.

The theory of normed spaces is often convenient in classical probability theory. However, if the systems of interests are in addition correlated with quantum systems, we have more structure available. A natural norm on quantum systems is the $\infty$-norm, the largest singular value. Hence we start by modeling a classical system as a normed space, and a quantum system as complex-valued matrices equipped with the $\infty$-norm. If we allow for correlations between the two, we have to define norms on their tensor product, fulfilling reasonable compatibility requirements. The framework of operator spaces axiomatizes such scenarios: an operator space is a normed space equipped with a sequence of norms describing possible correlations to quantum systems. If we now study linear maps between normed spaces, we can naturally consider these maps to be maps between operator spaces by letting them act trivially on the quantum part. Of course, the norm of the linear maps might change, since we now also allow for correlations to the quantum part (at the input as well as at the output). The associated norm, defined as the supremum with respect to quantum systems of any dimension, is called the completely bounded norm, or just cb-norm.

From this discussion, it is reasonable to expect that the property of being quantum-proof can be modeled as a cbnorm constraint. Indeed, we have that there exists operator space structures defined on the normed spaces described in Proposition II.1 such that the corresponding completely bounded norm

$$
\left\|\Delta[\mathrm{Ext}]: \cap \cdot \cap\left\{2^{k} \ell_{\infty}, \ell_{1}\right\} \rightarrow \ell_{1}\right\|_{\mathrm{cb}} \equiv\|\Delta[\mathrm{Ext}]\|_{\mathrm{cb}, \cap \cdot \cap\left\{2^{k} \ell_{\infty}, \ell_{1}\right\} \rightarrow \ell_{1}}
$$

captures the property of being quantum-proof.

Theorem II.2. Let Ext $=\left\{f_{s}\right\}_{s \in D}$ and $\Delta[$ Ext $]$ as defined in (17). Then, we have

$$
\begin{aligned}
\| \Delta[\text { Ext }]: \cap \cdot \cap\left\{2^{k} \ell_{\infty}, \ell_{1}\right\} \rightarrow \ell_{1} \|_{\mathrm{cb}} \leq \varepsilon & \Rightarrow \text { Ext is a quantum-proof }(k, 2 \varepsilon) \text { - extractor } \\
\text { Ext is a quantum-proof }(k-1, \varepsilon) \text {-extractor } & \Rightarrow \| \Delta[\text { Ext }]: \cap \cdot \cap\left\{2^{k} \ell_{\infty}, \ell_{1}\right\} \rightarrow \ell_{1} \|_{\mathrm{cb}} \leq 8 \varepsilon .
\end{aligned}
$$

Again the special case of strong extractors just follows by replacing $\Delta[$ Ext $]$ with $\Delta[\text { Ext }]_{S}$ in Theorem II.2. We note that we use the notation $\cap \cdot \cap\left\{2^{k} \ell_{\infty}, \ell_{1}\right\}$ for the operator space corresponding to the norm $\cap\left\{2^{k} \ell_{\infty}, \ell_{1}\right\}$. The reason is that there is a natural operator space associated with the norm $\cap\left\{2^{k} \ell_{\infty}, \ell_{1}\right\}$ for which one would use the same name, but the norm we consider here is slightly different.

\section{Applications}

We conclude that the ratio between the bounded norm $\cap\left\{2^{k} \ell_{\infty}, \ell_{1}\right\} \rightarrow \ell_{1}$ and its completely bounded extension in Theorem II.2 can be used to quantify to what extent extractors are quantum-proof. This type of comparison is of 
course very well studied in the theory of operator spaces. As a first straightforward application, we can use dimension dependent bounds for the maximal possible ratios between the completely bounded norm and the bounded norm,

$$
\|\cdot\|_{\mathrm{cb}, \cap \cdot \cap\left\{2^{k} \ell_{\infty}, \ell_{1}\right\} \rightarrow \ell_{1}} \leq \sqrt{2^{m}}\|\cdot\|_{\cap\left\{2^{k} \ell_{\infty}, \ell_{1}\right\} \rightarrow \ell_{1}}
$$

and with Proposition II.1 and Theorem II.2 we find that every $(k, \varepsilon)$-extractor is a quantum-proof $\left(k+1,6 \sqrt{2^{m}} \varepsilon\right)$ extractor. ${ }^{4}$ If $M$ is small this bound is non-trivial for weak extractors, but for strong extractors (for which the seed $D$ is part of the output $M=D \times M^{\prime}$ ) the bound becomes useless. However, using an operator version of the Cauchy-Schwarz inequality due to Haagerup we find the following bound for strong extractors.

Theorem II.3. Let Ext $=\left\{f_{s}\right\}_{s \in D}$, and $\Delta[\text { Ext }]_{S}$ as defined in (22). Then, we have

$$
\left\|\Delta[\mathrm{Ext}]_{S}: \cap\left\{2^{k} \ell_{\infty}, \ell_{1}\right\} \rightarrow \ell_{1}\right\| \leq \varepsilon \Rightarrow\left\|\Delta[\mathrm{Ext}]_{S}: \cap \cdot \cap\left\{2^{k+\log (1 / \varepsilon)} \ell_{\infty}, \ell_{1}\right\} \rightarrow \ell_{1}\right\|_{\mathrm{cb}} \leq 4 \sqrt{2^{m^{\prime}}} \sqrt{2 \varepsilon} .
$$

We get by Proposition II.1 that every strong $(k, \varepsilon)$-extractor is a quantum-proof strong $\left(k+\log (2 / \varepsilon), 12 \sqrt{2^{m^{\prime}}} \sqrt{2 \varepsilon}\right)$ extractor. This extends the result of König and Terhal for $m^{\prime}=1$ [23, Theorem 1] to arbitrary output sizes. The bound is useful as long as the output size is small but does of course not match Ta-Shma's conjecture that asks for an error dependence of $O\left(m^{\prime} \sqrt{\varepsilon}\right)$.

We can also analyze the simpler bounded norm $2^{k} \ell_{\infty} \rightarrow \ell_{1}$ instead of $\cap\left\{2^{k} \ell_{\infty}, \ell_{1}\right\} \rightarrow \ell_{1}$. Grothendieck's inequality [29, Corollary 14.2] then shows that the ratio between the bounded norm $2^{k} \ell_{\infty} \rightarrow \ell_{1}$ and its completely bounded extension is at most Grothendieck's constant $K_{G}<1.8$ :

$$
\|\cdot\|_{\mathrm{cb}, 2^{k} \ell_{\infty} \rightarrow \ell_{1}} \leq K_{G}\|\cdot\|_{2^{k} \ell_{\infty} \rightarrow \ell_{1}} .
$$

This gives the following bound. ${ }^{5}$

Theorem II.4. Let Ext $=\left\{f_{s}\right\}_{s \in D}$ and $\Delta[$ Ext $]$ as defined in (17). Then, we have

$$
\| \Delta[\text { Ext }]: \cap\left\{2^{k} \ell_{\infty}, \ell_{1}\right\} \rightarrow \ell_{1}\|\leq \varepsilon \Rightarrow\| \Delta[\text { Ext }]: \cap \cdot \cap\left\{2^{k} \ell_{\infty}, \ell_{1}\right\} \rightarrow \ell_{1} \|_{\mathrm{cb}} \leq K_{G} 2^{n-k} \varepsilon .
$$

Hence, we get by Proposition II.1 and Theorem II.1 that every $(k, \varepsilon)$-extractor is a quantum-proof $\left(k+1,6 K_{G} 2^{n-k} \varepsilon\right)$ extractor. This applies to weak and strong extractors equally since the statement is independent of the output size. So in particular if the input min-entropy is very high, e.g., $k \geq n-\log n$, we get a quantum-proof $\left(k+1,6 K_{G} n \varepsilon\right)$ extractor. Hence, very high min-entropy extractors are always (approximately) quantum-proof. ${ }^{6}$ We emphasize again that Theorem II.3 and Theorem II.4 do only make use of the definition of extractors and not of any potential structure of extractor constructions.

\begin{tabular}{l|lll} 
Strong $(k, \varepsilon)$-extractor & Quantum-proof & with parameters \\
\hline \hline Probabilistic constructions & $?$ & & \\
\hline Spectral (e.g., two-universal hashing) & $\checkmark$ & {$[4$, Thm. 4] } & $(k, c \sqrt{\varepsilon})$ \\
\hline Trevisan based & $\checkmark$ & {$[12$, Thm. 4.6] } & $(k+\log (1 / \varepsilon), c \sqrt{\varepsilon})$ \\
\hline One-bit output & $\checkmark$ & {$[23$, Thm. 1$]$} & $(k+\log (1 / \varepsilon), c \sqrt{\varepsilon})$ \\
\hline Small output & $\checkmark$ & [Thm. II.3] & $\left(k+\log (2 / \varepsilon), c \sqrt{2^{m}} \sqrt{2 \varepsilon}\right)$ \\
\hline High entropy & $\checkmark$ & {$[$ Thm. II.4] } & $\left(k+1, c 2^{n-k} \varepsilon\right)$ \\
\hline
\end{tabular}

TABLE I. Stability results for strong extractors: input $N=2^{n}$, output $M=2^{m}$, seed $D=2^{d}$, min-entropy $k$, error parameter $\varepsilon$, and $c$ represents possibly different constants.

\footnotetext{
4 We should point out that showing a similar bound, where the quantum error is upper bounded by $2^{m}$ multiplied by the classical error, can be obtained with a basic argument (not making use of any operator space theory).

${ }^{5}$ Interestingly this also implies that extractors for non-normalized inputs are automatically quantum-proof (e.g., spectral extractors [4]), and hence that the $\ell_{1}$-normalization condition on the input is crucial for studying to what extent extractors are quantum-proof.

${ }^{6}$ This result tightly matches with that the extractor construction of Gavinsky et al. [16]. In fact, their construction is an extractor for $k \geq n-n^{c}$ with error $\varepsilon=n^{-c^{\prime}}$ for some constants $c, c^{\prime}$ and it fails to be quantum-proof even if $k=n-O(\log n)$ with constant $\varepsilon$. Theorem II.4 says is that if the error in the classical case was a slightly smaller, for example super-polynomially small, then the extractor would have been quantum-proof.
} 


\section{B. Condensers}

The framework of normed spaces and operator spaces also allows to analyze to what extent condensers are quantumproof. Analogously as for extractors, we associate to a condenser Con $=\left\{f_{s}\right\}_{s \in D}$ a linear map from $N$ to $M$ given by

$$
[\mathrm{Con}](P):=\frac{1}{D} \cdot \sum_{s=1}^{D} F_{s}\left(P_{N}\right)
$$

The input constraint for condenser is the same as for extractors and in order to characterize the output constraint (12) we define the norm

$$
\left\|P_{N}\right\|_{\Sigma\left\{2^{k^{\prime}} \ell_{\infty}, \ell_{1}\right\}}:=\inf \left\{2^{k^{\prime}}\left\|Q_{1}\right\|_{\ell_{\infty}}+\left\|Q_{2}\right\|_{\ell_{1}}: Q_{1}+Q_{2}=P_{N}\right\}
$$

The bounded norm

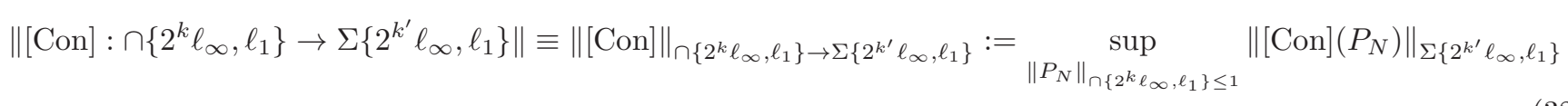

then gives the following norm characterization for condensers as shown in the following proposition.

Proposition II.5. Let Con $=\left\{f_{s}\right\}_{s \in D}$ and [Con] as defined in (30). Then, we have

$$
\begin{gathered}
\|[\text { Con }]: \cap\left\{2^{k} \ell_{\infty}, \ell_{1}\right\} \rightarrow \Sigma\left\{2^{k^{\prime}} \ell_{\infty}, \ell_{1}\right\} \| \leq \varepsilon \quad \Rightarrow \quad \text { Con is a }\left(k \rightarrow_{\varepsilon} k^{\prime}+\log (1 / \varepsilon)\right) \text { - condenser } \\
\text { Con is a }\left(k-1 \rightarrow_{\varepsilon} k^{\prime}+\log (1 / \varepsilon)\right) \text { - condenser } \quad \Rightarrow \quad \|[\text { Con }]: \cap\left\{2^{k} \ell_{\infty}, \ell_{1}\right\} \rightarrow \Sigma\left\{2^{k^{\prime}} \ell_{\infty}, \ell_{1}\right\} \| \leq 6 \varepsilon .
\end{gathered}
$$

Note that strong condensers are covered by this as a special case. To make this explicit we associate to a strong condenser Con $=\left\{f_{s}\right\}_{s \in D}$ the linear map

$$
[\mathrm{Con}]_{S}(P):=\frac{1}{D} \cdot \sum_{s=1}^{D} F_{s}\left(P_{N}\right) \otimes|s\rangle\left\langle\left. s\right|_{D}\right.
$$

and the corresponding statement can then be read off by replacing [Con] with [Con $]_{S}$ in Proposition II.5. Again we have that there exists operator space structures defined on the normed spaces described in Proposition II.5 such that the corresponding completely bounded norm

$$
\left\|[\mathrm{Con}]: \cap \cdot \cap\left\{2^{k} \ell_{\infty}, \ell_{1}\right\} \rightarrow \Sigma \cdot \Sigma\left\{2^{k^{\prime}} \ell_{\infty}, \ell_{1}\right\}\right\|_{\mathrm{cb}} \equiv\|[\mathrm{Con}]\|_{\mathrm{cb}, \cap \cdot \cap\left\{2^{k} \ell_{\infty}, \ell_{1}\right\} \rightarrow \Sigma \cdot \Sigma\left\{2^{k^{\prime}} \ell_{\infty}, \ell_{1}\right\}}
$$

captures the property of being quantum-proof.

Theorem II.6. Let Con $=\left\{f_{s}\right\}_{s \in D}$ and $[\mathrm{Con}]$ as defined in (30). Then, we have

$$
\|[\text { Con }]: \cap \cdot \cap\left\{2^{k} \ell_{\infty}, \ell_{1}\right\} \rightarrow \Sigma \cdot \Sigma\left\{2^{k^{\prime}} \ell_{\infty}, \ell_{1}\right\} \|_{\mathrm{cb}} \leq \frac{\varepsilon}{4} \Rightarrow \text { Con is a quantum-proof }\left(k \rightarrow{ }_{\varepsilon} k^{\prime}+\log (1 / \varepsilon)\right)-\operatorname{condenser}
$$

Con is a quantum-proof $\left(k-1 \rightarrow_{\varepsilon} k^{\prime}+\log (1 / \varepsilon)\right)$ - condenser $\Rightarrow \quad \|[$ Con $]: \cap \cdot \cap\left\{2^{k} \ell_{\infty}, \ell_{1}\right\} \rightarrow \Sigma \cdot \Sigma\left\{2^{k^{\prime}} \ell_{\infty}, \ell_{1}\right\} \|_{\mathrm{cb}} \leq 8 \varepsilon$.

The special case of strong condensers just follows by replacing [Con] with [Con $]_{S}$ in Theorem II.6. Note that even though an extractor is just a condenser with full output entropy $k^{\prime}=m$, our norm characterization for condensers can not directly be used to characterize extractors because there is a $\operatorname{loss}$ of $\log (1 / \varepsilon)$ for the output entropy $k^{\prime}$ (see Proposition II.5 and Theorem II.6). However, for that reason we have the separate norm characterization for extractors in Section II A. 


\section{Applications}

It is known that condensers are closely related to graph-theoretic problems [39], and here we make exactly such a connection (that is different from previously studied connections). Using the bounded norm characterization in Proposition II.5, we can show that evaluating the performance of a condenser corresponds to an instance of a well studied combinatorial problem, called bipartite densest subgraph. For this we think of condensers as bipartite graphs $G=(N, M, V \subset N \times M, D)$ with left index set $N$, right index set $M$, edge set $V$ having left degree $D$, and neighbor function $\Gamma: N \times D \rightarrow M$. The identification with the usual definition of condensers Con $=\left\{f_{s}\right\}_{s \in D}$ is just by setting

$$
\Gamma(\cdot, s):=f_{s}(\cdot)
$$

for every value of the seed $s \in D$.

Proposition II.7. Let $\mathrm{Con}=\left\{f_{s}\right\}_{s \in D}$, [Con] as defined in (30), $G=(N, M, V, D)$ be the bipartite graph as defined in (39), and Dense $\left(G, 2^{k}, 2^{k^{\prime}}\right)$ be the optimal value of the quadratic program

$$
\begin{aligned}
\operatorname{Dense}\left(G, 2^{k}, 2^{k^{\prime}}\right):=\text { maximize } & \sum_{(x, y) \in V} f_{x} g_{y} \\
\text { subject to } & f_{x}, g_{y} \in\{0,1\} \\
& \sum_{x} f_{x} \leq 2^{k} \\
& \sum_{y} g_{y} \leq 2^{k^{\prime}} .
\end{aligned}
$$

Then, we have

$$
\|[\text { Con }]: \cap\left\{2^{k} \ell_{\infty}, \ell_{1}\right\} \rightarrow \Sigma\left\{2^{k^{\prime}} \ell_{\infty}, \ell_{1}\right\} \| \leq \varepsilon \quad \Leftrightarrow \quad \operatorname{Dense}\left(G, 2^{k}, 2^{k^{\prime}}\right) \leq 2^{k+d} \varepsilon .
$$

Hence, we try to find the subgraph of $G$ with the biggest number of edges, having $2^{k}$ vertices on the left and $2^{k^{\prime}}$ vertices on the right. The norm condition for being a condenser (Proposition II.5) then just says that the size of the edge set of all such subgraphs has to be bounded by $2^{k} D \varepsilon$. This is exactly an instance of the bipartite densest $\left(2^{k}, 2^{k^{\prime}}\right.$-subgraph problem. The (bipartite) densest subgraph problem is well studied in theoretical computer science, but its hardness remains elusive. However, it is known that usual semidefinite program (SDP) relaxation possess a large integrality gap for random graphs $[7,15]$. Interestingly, we can show that the densest subgraph SDP relaxations are not only an upper bound on the quadratic program (40) characterizing characterizing the bounded norm of condensers, but also on the completely bounded norm of condensers.

Seeing condensers as bipartite graphs also allows us to define a Bell inequality (two-player game) such that the classical value characterizes the condenser property and the entangled value characterizes the quantum-proof condenser property (see [9] for a review article about Bell inequalities / two-player games). Starting from the bipartite graph $G=(N, M, V, D)$ as defined in (39), we use operator space techniques by Junge [17] to define a two-player game $\left(G ; 2^{k}, 2^{k^{\prime}}\right)$ with classical value $\omega\left(G ; 2^{k}, 2^{k^{\prime}}\right)$ and entangled value $\omega^{*}\left(G ; 2^{k}, 2^{k^{\prime}}\right)$, that has the following properties.

Theorem II.8. Let Con $=\left\{f_{s}\right\}_{s \in D}$, [Con] as defined in (30), $G=(N, M, V, D)$ be the bipartite graph as defined in (39), and $\left(G ; 2^{k}, 2^{k^{\prime}}\right)$ be the two-player game defined in Section VID. Then, there is a constant $c>0$ such that

$$
\omega\left(G ; 2^{k}, 2^{k^{\prime}}\right) \leq 2^{-k^{\prime}} \cdot \|[\text { Con }]: \cap\left\{2^{k} \ell_{\infty}, \ell_{1}\right\} \rightarrow \Sigma\left\{2^{k^{\prime}} \ell_{\infty}, \ell_{1}\right\} \| \leq c \cdot \omega\left(G ; 2^{k}, 2^{k^{\prime}}\right),
$$

as well as

$$
\omega^{*}\left(G ; 2^{k}, 2^{k^{\prime}}\right) \leq 2^{-k^{\prime}} \cdot \|[\text { Con }]: \cap \cdot \cap\left\{2^{k} \ell_{\infty}, \ell_{1}\right\} \rightarrow \Sigma \cdot \Sigma\left\{2^{k^{\prime}} \ell_{\infty}, \ell_{1}\right\} \|_{\mathrm{cb}} \leq c \cdot \omega^{*}\left(G ; 2^{k}, 2^{k^{\prime}}\right) .
$$

Furthermore, the amount of entanglement used by the players corresponds to the dimension of the quantum side information system $Q$.

Theorem II.8 shows that for every condenser construction there is a corresponding Bell inequality, and that the degree to which this inequality is violated by quantum mechanics characterizes how quantum-proof the condenser construction is (and vice versa). So in particular, fully quantum-proof condensers (which includes quantum-proof extractors) have a corresponding Bell inequality that is not violated by quantum mechanics. 


\section{OPEN PROBLEMS}

We showed how the theory of operator spaces provides a useful framework for studying the behavior of randomness extractors and condensers in the presence of quantum adversaries. However, there are many questions left open from here and we believe that the following are particularly interesting to explore:

- The main question if there exist good classical extractors that are not quantum-proof with a large gap still remains open. More precisely we would like to understand the classical/quantum separation better by finding tighter upper bounds (as in Theorem II.3 and Theorem II.4) as well as tighter lower bounds (as in [16]) on the size of the gap.

- Is it possible to give an upper bound on the dimension of the quantum adversary that is sufficient to consider? This is also a natural question in the norm formulation (Theorem II.2 and Theorem II.6) and the Bell inequality formulation (Theorem II.8). In the first case it translates into the question for what dimension the completely bounded norm saturates, and in the latter case it translates into the question how much entanglement is needed to achieve the optimal entangled value.

- Given our new connection to Bell inequalities (Theorem V.6) it would be interesting to explore the corresponding Bell inequalities of quantum-proof extractor constructions (since they can not be violated by quantum mechanics).

- What other explicit extractor constructions are quantum-proof? This includes variations of Trevisan's constructions as, e.g., listed in [12, Section 6], but also condenser based constructions [31, 32]. Here, the motivation is that all of these constructions have better parameters than any known quantum-proof construction.

- Operator space techniques might also be useful for analyzing fully quantum and quantum-to-classical randomness extractors as described in [4-6].

\section{PRELIMINARIES}

\section{A. Quantum information}

In quantum theory, a system is described by an inner-product space, that we denote here by letters like $N, M, Q .{ }^{7}$ Note that we use the same symbol $Q$ to label the system, the corresponding inner-product space and also the dimension of the space. Let $\operatorname{Mat}_{Q}(S)$ be the vector space of $Q \times Q$ matrices with entries in $S$. Whenever $S$ is not specified, it is assumed to be the set of complex numbers $\mathbb{C}$, i.e., we write $\operatorname{Mat}_{Q}(\mathbb{C})=: \operatorname{Mat}_{Q}$. The state of a system is defined by a positive semidefinite operator $\rho_{Q}$ with trace 1 acting on $Q$. The set of states on system $Q$ is denoted by $\mathcal{S}(Q) \subset \operatorname{Mat}_{Q}(\mathbb{C})$. The inner-product space of a composite system $Q N$ is given by the tensor product of the inner-product spaces $Q \otimes N=: Q N$. From a joint state $\rho_{Q N} \in \mathcal{S}(Q N)$, we can obtain marginals on the system $Q$ by performing a partial trace of the $N$ system $\rho_{Q}:=\operatorname{Tr}_{N}\left[\rho_{Q N}\right]$. A state $\rho_{Q N}$ on $Q N$ is called quantum-classical (with respect to some basis) if it can be written as $\rho_{Q N}=\sum_{x} \rho_{x} \otimes|x\rangle\langle x|$ for some basis $\{|x\rangle\}$ of $N$ and some positive semidefinite operators $\rho_{x}$ acting on $Q$. We denote the maximally mixed state on system $N$ by $v_{N}$.

To measure the distance between two states, we use the trace norm $\|A\|_{1}:=\operatorname{Tr}\left[\sqrt{A^{*} A}\right]$, where $A^{*}$ is the conjugate transpose of $A$. In the special case when $A$ is diagonal, $\|A\|_{1}$ becomes the familiar $\ell_{1}$ norm of the diagonal entries. Moreover, the Hilbert-Schmidt norm is defined as $\|A\|_{2}:=\sqrt{\operatorname{Tr}\left[A^{*} A\right]}$, and when $A$ is diagonal this becomes the usual $\ell_{2}$ norm. Another important norm we use is the operator norm, or the largest singular value of $A$, denoted by $\|A\|_{\infty}$. When $A$ is diagonal, this corresponds to the familiar $\ell_{\infty}$ norm of the diagonal entries. For a probability distribution $P_{N} \in \mathcal{S}(N),\left\|P_{N}\right\|_{\ell_{\infty}}$ corresponds to the optimal probability with which $P_{N}$ can be guessed. We write

$$
H_{\min }(N)_{P}:=-\log \left\|P_{N}\right\|_{\ell_{\infty}},
$$

the min-entropy of $P_{N}$. More generally, the conditional min-entropy of $N$ given $Q$ is used to quantify the uncertainty in the system $N$ given the system $Q$. The conditional min-entropy is defined as

$$
H_{\min }(N \mid Q)_{\rho}:=-\log \min _{\sigma_{Q} \in \mathcal{S}(Q)}\left\|\left(\mathbb{1}_{N} \otimes \sigma_{Q}^{-1 / 2}\right) \rho_{N Q}\left(\mathbb{1}_{N} \otimes \sigma_{Q}^{-1 / 2}\right)\right\|_{(\infty ; \infty)},
$$

\footnotetext{
${ }^{7}$ In the following all spaces are assumed to be finite-dimensional.
} 
with generalized inverses, and $\|\cdot\|_{(\infty ; \infty)}$ the operator norm on $\operatorname{Mat}_{Q} \otimes \operatorname{Mat}_{N}=: \operatorname{Mat}_{Q N}$. Note that in the special case where the system $Q$ is trivial, we have $H_{\min }(N)_{\rho}=-\log \left\|\rho_{N}\right\|_{\infty}$. In fact, the general case also corresponds to a norm, we have

$$
H_{\min }(N \mid Q)_{\rho}=-\log \left\|\rho_{Q N}\right\|_{(1 ; \infty)},
$$

where the norm $\|\cdot\|_{(1 ; \infty)}$ on Mat $_{Q N}$ is defined as

$$
\|A\|_{(1 ; \infty)}:=\inf \left\{\left\|B_{1}\right\|_{2}\|C\|_{(\infty ; \infty)}\left\|B_{2}\right\|_{2}: A=\left(B_{1} \otimes \mathbb{1}_{N}\right) C\left(B_{2} \otimes \mathbb{1}_{N}\right) ; B_{1}, B_{2} \in \operatorname{Mat}_{Q}\right\} .
$$

A proof of this is given in the Appendix as Proposition A.3.

\section{B. Normed spaces}

A vector space $E$ together with a norm $\|\cdot\|_{E}$ defines a normed space denoted by $\left(E,\|\cdot\|_{E}\right)$. On the dual space $E^{*}$ the dual norm $\|\cdot\|_{E^{*}}$ is defined as

$$
\|f\|_{E^{*}}:=\sup _{\|e\|_{E} \leq 1}|f(e)|,
$$

and hence $\left(E^{*},\|\cdot\|_{E^{*}}\right)$ is again a normed space. We have that $E$ is isomorphic to $E^{* *}$ since we restrict to finitedimensional spaces.

The vector space of linear operators from $E$ to $F$ is denoted by $\operatorname{Lin}(E, F)$ and for normed spaces $E$ and $F$ there is a natural norm induced on $\operatorname{Lin}(E, F)$ defined by

$$
\|u\|_{E \rightarrow F}:=\sup _{\|x\|_{E} \leq 1}\|u(x)\|_{F},
$$

where $u \in \operatorname{Lin}(E, F)$. This norm is called the bounded norm and we also use the notation

$$
\|u: E \rightarrow F\|:=\|u\|_{E \rightarrow F} .
$$

For the corresponding normed space we write $B(E, F):=\left(\operatorname{Lin}(E, F),\|\cdot\|_{E \rightarrow F}\right)$. Note that every $u: E \rightarrow F$ has finite bounded norm (since we restrict to finite-dimensional spaces). The dual of the $\|\cdot\|_{E \rightarrow F}$ norm is the $\|\cdot\|_{F^{*} \rightarrow E^{*}}$ norm. We define the adjoint map $u^{*}: F^{*} \rightarrow E^{*}$ as $\left(u^{*}\left(f^{*}\right)\right)(e)=f^{*}(u(e))$ for $e \in E$ and $f^{*} \in F^{*}$. It is simple to check that we have

$$
\|u\|_{E \rightarrow F}=\left\|u^{*}\right\|_{F^{*} \rightarrow E^{*}} .
$$

The norms we are interested in are constructed by combining the $\ell_{\infty}$ norm and the $\ell_{1}$ norm. The following gives a general way of combining two norms. For $\|\cdot\|_{\alpha}$ and $\|\cdot\|_{\beta}$ norms on a vector space $E$, we define the $\cap$-norm as

$$
\|x\|_{\cap\{\alpha, \beta\}}:=\max \left\{\|x\|_{\alpha},\|x\|_{\beta}\right\},
$$

where $x \in E$. The dual norm then takes the following form.

Proposition IV.1. For the norm $\|\cdot\|_{\cap\{\alpha, \beta\}}$ on a vector space $E$, the corresponding dual norm $\|\cdot\|_{\Sigma\left\{\alpha^{*}, \beta^{*}\right\}}$ on the dual space $E^{*}$ takes the form

$$
\|y\|_{\Sigma\left\{\alpha^{*}, \beta^{*}\right\}}=\inf \left\{\left\|y_{1}\right\|_{\alpha^{*}}+\left\|y_{2}\right\|_{\beta^{*}}: y_{1}+y_{2}=y\right\}
$$

where $y \in E^{*}$.

Proof. For a given $y \in E^{*}$, we write the dual convex program to $\|y\|_{\Sigma\left\{\alpha^{*}, \beta^{*}\right\}}=\inf \left\{\left\|y_{1}\right\|_{\alpha^{*}}+\left\|y_{2}\right\|_{\beta^{*}}: y=y_{1}+y_{2}\right\}$ as

$$
\sup _{x \in E} \inf _{y_{1} y_{2}}\left\|y_{1}\right\|_{\alpha^{*}}+\left\|y_{2}\right\|_{\beta^{*}}+\left(y-y_{1}-y_{2}\right)(x) .
$$

But observe that $\inf _{y_{1}}\left\|y_{1}\right\|_{\alpha^{*}}-y_{1}(x)=0$ if $\|x\|_{\alpha}=\sup _{y_{1}}\left\{y_{1}(x):\left\|y_{1}\right\|_{\alpha^{*}} \leq 1\right\} \leq 1$ and $\inf _{y_{1}}\left\|y_{1}\right\|_{\alpha^{*}}-y_{1}(x)=-\infty$ otherwise. Thus the dual convex program can be written as

$$
\sup _{x \in E}\left\{y(x):\|x\|_{\alpha} \leq 1,\|x\|_{\beta} \leq 1\right\},
$$

which is the definition of the dual norm of the norm $\|\cdot\|_{\cap\{\alpha, \beta\}}$. We conclude by observing that strong duality holds in this case. 


\section{Operator spaces}

For the convenience of the reader we recall a few basic facts about the theory of operator spaces, sometimes also referred to as "quantized Banach spaces". For a more in depth treatment we refer the reader to Pisier's book [28]. An operator space is a normed space $\left(E,\|\cdot\|_{E}\right)$ together with a sequence of norms $\|\cdot\|_{M_{Q}(E)}$ on the spaces Mat $(E)$ satisfying the following consistency conditions for all $Q \geq 2$ (also $\left.\|\cdot\|_{E}=\|\cdot\|_{M_{1}(E)}\right)$.

Definition IV.1 (Operator space). An (abstract) operator space is a vector space E together with a sequence of norms $\|\cdot\|_{M_{Q}(E)}$ on $\operatorname{Mat}_{Q}(E)$ such that for $Q \in \mathbb{N}$ :

1. For all $x \in \operatorname{Mat}_{Q}(E)$ and $x^{\prime} \in \operatorname{Mat}_{Q}(E)$,

$$
\left\|\left(\begin{array}{cc}
x & 0 \\
0 & x^{\prime}
\end{array}\right)\right\|_{M_{Q+Q^{\prime}}(E)}=\max \left\{\|x\|_{M_{Q}(E)},\left\|x^{\prime}\right\|_{M_{Q^{\prime}}(E)}\right\} .
$$

2. For all $\alpha, \beta \in \operatorname{Mat}_{Q}$ and $x \in \operatorname{Mat}_{Q}(E)$,

$$
\|\alpha \cdot x \cdot \beta\|_{M_{Q}(E)} \leq\|\alpha\|_{\infty}\|x\|_{M_{Q}(E)}\|\beta\|_{\infty},
$$

where the notation $\alpha \cdot x$ refers to usual multiplication of matrices.

We write $\left(E,\|\cdot\|_{M_{Q}(E)}\right)$ for the operator space structure.

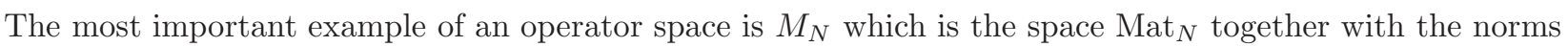

$$
\|\cdot\|_{M_{Q}\left(M_{N}\right)}:=\|\cdot\|_{(\infty ; \infty)}
$$

the usual operator norms on Mat $_{Q N}$. It is easy to verify that the two conditions (59) and (60) are satisfied. Alternatively we could also define an operator space (concrete operator space) on a normed space $\left(E,\|\cdot\|_{E}\right)$ by seeing $\left(E,\|\cdot\|_{E}\right)$ as a subspace of $\left(\operatorname{Mat}_{N},\|\cdot\|_{\infty}\right)$ and the norms $\|\cdot\|_{M_{Q}(E)}$ induced by the operator norms $\|\cdot\|_{(\infty ; \infty)}$ on $\operatorname{Mat}_{Q N}$.

Since Ruan [34] proved that every abstract operator space can be realized as a concrete operator space (see also [28, Chapter 2.2]) these two definitions are really equivalent. ${ }^{8}$ For example, consider the $\operatorname{subspace}^{\text {Diag }}{ }_{N} \subset$ Mat $_{N}$ of diagonal matrices. It is then immediate to deduce that for this concrete operator space $E$, the norm $\|\cdot\|_{M_{1}(E)}$ is simply the $\ell_{\infty}$ norm of the diagonal vector. As another example, consider the subspace $C_{N}\left(R_{N}\right)$ of matrices such that only the first column (row) contains non-zero elements. This defines a concrete operator space on $\mathbb{C}^{N}$, the norm $M_{1}\left(C_{N}\right)=M_{1}\left(R_{N}\right)$ is simply the $\ell_{2}$ norm of the vector. Note that even though as normed spaces $C_{N}$ and $R_{N}$ are both isomorphic to $\left(\mathbb{C}^{N}, \ell_{2}\right)$, the aforementioned operator space structures are different. In fact, a given normed space has in general many possible operator space structures. However, for many normed spaces that we are interested in, there is one "natural" operator space structure. For example, for the normed space (Mat $\left.{ }_{N},\|\cdot\|_{\infty}\right)$ there is the natural operator space structure $M_{N}$ defined above. Also, for the normed space $\left(\right.$ Mat $_{N},\|\cdot\|_{1}$ ) there is a natural operator space structure as the operator space dual of $M_{N}$. We will discuss this in detail after the definition of the operator space dual (Definition IV.3).

The bounded norm as in (52) is fundamental in understanding linear maps between normed spaces. The analogous norm for linear maps between operator spaces is the completely bounded norm (because completely bounded maps are the morphisms in the category of operator spaces). In the quantum information literature, the completely bounded norm usually refers specifically to maps from the operator space $M_{N}$ to $M_{M}$. The dual norm is called the diamond norm, first introduced in quantum information by Kitaev [21]. Here we are concerned with the completely bounded norm between more general operator spaces.

Definition IV.2 (Completely bounded norm). For operator spaces $E$ and $F$ the completely bounded norm of $u \in$ $\operatorname{Lin}(E, F)$ is defined as

$$
\|u\|_{\mathrm{cb}, E \rightarrow F}:=\sup _{Q}\left\|u_{Q}\right\|_{M_{Q}(E) \rightarrow M_{Q}(F)},
$$

where for $\left\{x_{i j}\right\}_{i j} \in \operatorname{Mat}_{Q}(E), u_{Q}\left(\left\{x_{i j}\right\}_{i j}\right):=\left\{u\left(x_{i j}\right)\right\}_{i j}$, or simply $u_{Q}=\mathbb{1}_{Q} \otimes u$. We also use the notation

$$
\|u: E \rightarrow F\|_{\mathrm{cb}}:=\|u\|_{\mathrm{cb}, E \rightarrow F} .
$$

\footnotetext{
${ }^{8}$ In general, one needs to embed $E$ into the space of bounded operators on an infinite dimensional Hilbert space.
} 
Hence $\left(\operatorname{Lin}(E, F),\|\cdot\|_{\mathrm{cb}, E \rightarrow F}\right)$ is also a normed space. Note that every $u: E \rightarrow F$ has finite completely bounded norm (since we restrict to finite-dimensional spaces), however $\|\cdot\|_{E \rightarrow F}$ is in general smaller than $\|\cdot\|_{\mathrm{cb}, E \rightarrow F}$. Later, we are interested in upper bounding this ratio for particular operator spaces and maps. For general $E, F$ and $u: E \rightarrow F$ of rank $M$ it is known that the ratio of the completely bounded to the bounded norm is at most $M / 2^{1 / 4}[28$, Theorem $7.15]$.

It is in general not true that we can restrict the supremum in (62) to finite $Q$. However, in case the that the target normed space is $M_{N}$, we have [28, Proposition 12] (or [44, Theorem 5])

$$
\|u\|_{\mathrm{cb}, E \rightarrow M_{N}}=\left\|u_{N}\right\|_{M_{N}(E) \rightarrow M_{N}\left(M_{N}\right)} .
$$

This raises the question whether there are specific operator space structures such that all bounded norms are also completely bounded. Such structures do in fact exist, and are called minimal and maximal operator space structures.

Theorem IV.2. [28, Chapter 3] Let $\left(E,\|\cdot\|_{E}\right)$ be a normed space. Then there exists two (in general different) operator spaces $\max (E)$ and $\min (E)$, such that we have for all operator spaces $F$ and $u \in \operatorname{Lin}(E, F)$ and $v \in \operatorname{Lin}(F, E)$,

$$
\|u\|_{\mathrm{cb}, F \rightarrow \min (E)}=\|u\|_{F \rightarrow \min (E)} \text { and }\|v\|_{\mathrm{cb}, \max (E) \rightarrow F}=\|v\|_{E \rightarrow F} .
$$

If $E$ and $F$ are operator spaces, then we can define a natural operator space structure on $\operatorname{Lin}(E, F)$ called $C B(E, F)$. To see this, observe that we can see $\operatorname{Mat}_{Q}(\operatorname{Lin}(E, F))$ as elements of $\operatorname{Lin}\left(E, \operatorname{Mat}_{Q}(F)\right)$. Thus for $x \in \operatorname{Mat}_{Q}(\operatorname{Lin}(E, F))$, we define

$$
\|x\|_{M_{Q}(C B(E, F))}:=\|x\|_{\mathrm{cb}, E \rightarrow \operatorname{Mat}_{Q}(F)} .
$$

It is then simple to verify the two conditions (59) and (60). By taking $F=\mathbb{C}$, this allows us to define the notion of a dual operator space.

Definition IV.3 (Operator space dual). For an operator space E, the dual operator space denoted $E^{*}$ is defined as

$$
\|x\|_{M_{Q}\left(E^{*}\right)}:=\|x\|_{\mathrm{cb}, E \rightarrow M_{Q}},
$$

where $x \in \operatorname{Mat}_{Q}\left(E^{*}\right)$ is viewed as an element of $\operatorname{Lin}\left(E, \operatorname{Mat}_{Q}\right)$.

For $u \in \operatorname{Lin}(F, E)$ we have for the adjoint $u^{*} \in \operatorname{Lin}\left(F^{*}, E^{*}\right)$ that $\|u\|_{\mathrm{cb}}=\left\|u^{*}\right\|_{\mathrm{cb}}$. We also have $E^{* *}=E$ with $M_{Q}\left(E^{* *}\right)=M_{Q}(E)$ since we restrict to finite-dimensional spaces. If we consider the norm for $Q=1$, then $\|\cdot\|_{E^{*}}$ corresponds to the dual norm of $\|\cdot\|_{E}$ (this follows since $\|\cdot\|_{\mathrm{cb}, E \rightarrow \mathbb{C}}=\|\cdot\|_{E \rightarrow \mathbb{C}}$ by, e.g., [28, Proposition 1.10]). However, this is not the case for $Q \geq 2$, that is, $M_{Q}\left(E^{*}\right)$ on $\operatorname{Mat}_{Q}\left(E^{*}\right)$ is in general not the dual norm of $M_{Q}(E)$ on $\operatorname{Mat}_{Q}(E)$.

As an example let us now consider the dual operator space of $M_{N}$, called the trace class operator space $S_{N}^{1}$.

Proposition IV.3. The sequence of norms for the trace class operator space $S_{N}^{1}$ are given by

$$
\|x\|_{M_{Q}\left(S_{N}^{1}\right)}=\|x\|_{(\infty ; 1)}:=\sup \left\{\left\|\left(A \otimes \mathbb{1}_{N}\right) x\left(B \otimes \mathbb{1}_{N}\right)\right\|_{(1 ; 1)}:\|A\|_{2},\|B\|_{2} \leq 1 ; A, B \in \operatorname{Mat}_{Q}\right\},
$$

where $x \in \operatorname{Mat}_{Q}\left(\operatorname{Mat}_{N}\right)$, and $\|\cdot\|_{(1 ; 1)}$ the trace norm on $\operatorname{Mat}_{Q N}$.

We observe that the norm $\|\cdot\|_{(\infty ; 1)}$ is the dual norm of the norm $\|\cdot\|_{(1 ; \infty)}$ characterizing the conditional minentropy (50); see Proposition A.2.

Proof. In order to compute the dual norm, we first explicitly map $x \in \operatorname{Mat}_{Q}\left(\right.$ Mat $\left._{N}\right)$ to an element of Lin(Mat $N$, Mat $\left.Q\right)$. For this, we see $x$ as the Choi matrix of a map $u \in \operatorname{Lin}\left(\operatorname{Mat}_{N}, \operatorname{Mat}_{Q}\right) \operatorname{defined~by~}$

$$
\operatorname{Tr}[d u(c)]=\operatorname{Tr}\left[x\left(d \otimes c^{T}\right)\right]
$$

for any $c \in \operatorname{Mat}_{N}, d \in \operatorname{Mat}_{Q}$, where $c^{T}$ denotes the transpose of $c$ in the standard basis. Using the definition of operator space dual (Definition IV.3), we have that

$$
\|x\|_{M_{Q}\left(S_{N}^{1}\right)}=\left\|u: M_{N} \rightarrow M_{Q}\right\|_{\mathrm{cb}}=\left\|u: M_{Q}\left(M_{N}\right) \rightarrow M_{Q}\left(M_{Q}\right)\right\|
$$

where we used (64) to simplify the completely bounded norm. Continuing we get,

$$
\begin{aligned}
\|x\|_{M_{Q}\left(S_{N}^{1}\right)} & =\sup \left\{\left\|\left(\mathbb{1}_{Q} \otimes u\right)(z)\right\|_{(\infty ; \infty)}: z \in \operatorname{Mat}_{Q N},\|z\|_{(\infty ; \infty)} \leq 1\right\} \\
& =\sup \left\{\operatorname{Tr}\left[\left(\mathbb{1}_{Q} \otimes u\right)(z) a b^{*}\right]: z \in \operatorname{Mat}_{Q N},\|z\|_{(\infty ; \infty)} \leq 1 ; a, b \in \mathbb{C}^{Q^{2}},\|a\|_{2},\|b\|_{2} \leq 1\right\} .
\end{aligned}
$$


A straightforward calculation (Lemma A.4) shows that

$$
\operatorname{Tr}\left[\left(\mathbb{1}_{Q} \otimes u\right)(z) a b^{*}\right]=\operatorname{Tr}\left[\left(\bar{B} \otimes \mathbb{1}_{N}\right) x\left(A^{T} \otimes \mathbb{1}_{N}\right) z^{T}\right],
$$

where $A:=\sum_{i j} a_{i j}|i\rangle\langle j|$ and $B:=\sum_{i j} b_{i j}|i\rangle\langle j|$. To conclude, we use that $\left\|z^{T}\right\|_{(\infty ; \infty)}=\|z\|_{(\infty ; \infty)},\left\|A^{T}\right\|_{2}=\|a\|_{2}$, $\|\bar{B}\|_{2}=\|b\|_{2}$, and that the trace norm is the dual norm of the operator norm.

We now can define the analogs of the $\cap$ - and $\Sigma$-norms for operator spaces. If $M_{Q}(\alpha)$ and $M_{Q}(\beta)$ denote two operator structures on the same vector space $V$, then we denote by $\|\cdot\|_{\alpha^{*}}^{(Q)}$ and $\|\cdot\|_{\beta^{*}}^{(Q)}$ the sequence of norms on the dual space $E^{*}$ giving rise to the operator space duals.

Definition IV.4 ( $\cap$-norm). Let $E$ and $F$ be two operator spaces on the same vector space $V$. Then we define a new operator space $\cap\{E, F\}$ by the sequence of norms

$$
\|x\|_{M_{Q}(\cap\{E, F\})}:=\max \left\{\|x\|_{M_{Q}(E)},\|x\|_{M_{Q}(F)}\right\} .
$$

The operator space dual of $\cap\{E, F\}$ is denoted $\Sigma\left\{E^{*}, F^{*}\right\}$.

One might now think that $M_{Q}\left(\Sigma\left\{E^{*}, F^{*}\right\}\right)$ is equal to the $\Sigma$-norm of the norms $M_{Q}\left(E^{*}\right)$ and $M_{Q}\left(F^{*}\right)$. This is almost the case but only up to a factor of 2 ; see [28, Chapter 2.7]. A more detailed discussion of the $\cap$ - and $\Sigma$-operator space structures can be found in [28, Chapter 2.7].

\section{EXTRACTORS}

\section{A. Extractor property as a bounded norm}

Here we characterize extractors in terms of the bounded norm $\cap\left\{2^{k} \ell_{\infty}, \ell_{1}\right\} \rightarrow \ell_{1}$.

Proposition V.1. Let Ext $=\left\{f_{s}\right\}_{s \in D}$ and $\Delta[\mathrm{Ext}]$ as defined in (17). Then, we have

$$
\begin{aligned}
\| \Delta[\text { Ext }]: \cap\left\{2^{k} \ell_{\infty}, \ell_{1}\right\} \rightarrow \ell_{1} \| \leq \varepsilon & \Rightarrow \text { Ext is a }(k, \varepsilon) \text { - extractor } \\
\text { Ext is a }(k-1, \varepsilon) \text { - extractor } & \Rightarrow \quad \| \Delta[\text { Ext }]: \cap\left\{2^{k} \ell_{\infty}, \ell_{1}\right\} \rightarrow \ell_{1} \| \leq 3 \varepsilon .
\end{aligned}
$$

Proof. We first prove (20). For any probability distribution $P$ with min-entropy at least $k$ we have $\|P\|_{\ell_{1}}=1$ as well as $\|P\|_{2^{k} \ell_{\infty}} \leq 1$. Hence, $\|P\|_{\cap\left(2^{k} \ell_{\infty}, \ell_{1}\right)} \leq 1$ and by the definition of the bounded norm this implies the claim $\|\Delta[\operatorname{Ext}](P)\|_{\ell_{1}} \leq \varepsilon$.

To prove (21) consider a distribution $P$ with $\|P\|_{\ell_{1}} \leq 1$ and $\|P\|_{\ell_{\infty}} \leq 2^{-k}$. Then let $P^{+}(x)=\max \{P(x), 0\}$ and $P^{-}(x)=\max \{-P(x), 0\}$, and note that $\left\|P^{+}\right\|_{\ell_{\infty}},\left\|P^{-}\right\|_{\ell_{\infty}} \leq 2^{-k}$. As the extractor property only applies for normalized distributions, we extend $P^{+}, P^{-}$into a probability distributions $\bar{P}^{+}=P^{+}+\left(1-\left\|P^{+}\right\|_{\ell_{1}}\right) v_{N}$ and $\bar{P}^{-}(x)=P^{-}(x)+\left(1-\left\|P^{-}\right\|_{\ell_{1}}\right) v_{N}$. Now observe that $\left\|\bar{P}^{+}\right\|_{\ell_{\infty}} \leq\|P\|_{\ell_{\infty}}+\frac{1}{N} \leq 2^{-(k-1)}$ and the similar bound holds for $\left\|\bar{P}^{-}\right\|_{\ell_{\infty}}$. Thus, we have

$$
\begin{aligned}
\|\Delta[\operatorname{Ext}](P)\|_{\ell_{1}} & =\left\|\Delta[\operatorname{Ext}]\left(P^{+}\right)-\Delta[\operatorname{Ext}]\left(P^{-}\right)\right\|_{\ell_{1}} \\
& \leq\left\|\Delta[\operatorname{Ext}]\left(P^{+}\right)\right\|_{\ell_{1}}+\left\|\Delta[\operatorname{Ext}]\left(P^{-}\right)\right\|_{\ell_{1}} \\
& \leq\left\|\Delta[\operatorname{Ext}]\left(\bar{P}^{+}\right)\right\|_{\ell_{1}}+\left(1-\left\|P^{+}\right\|_{\ell_{1}}\right)\left\|\Delta[\operatorname{Ext}]\left(v_{N}\right)\right\|_{\ell_{1}}+\left\|\Delta[\operatorname{Ext}]\left(\bar{P}^{-}\right)\right\|_{\ell_{1}}+\left(1-\left\|P^{-}\right\|_{\ell_{1}}\right)\left\|\Delta[\operatorname{Ext}]\left(v_{N}\right)\right\|_{\ell_{1}} \\
& \leq\left\|\Delta[\operatorname{Ext}]\left(\bar{P}^{+}\right)\right\|_{\ell_{1}}+\left\|\Delta[\operatorname{Ext}]\left(\bar{P}^{-}\right)\right\|_{\ell_{1}}+\left\|\Delta[\operatorname{Ext}]\left(v_{N}\right)\right\|_{\ell_{1}} \\
& \leq 3 \varepsilon
\end{aligned}
$$

where we used the fact that $\bar{P}^{+}, \bar{P}^{-}$and $v_{N}$ have min-entropy at least $k-1$.

\section{B. Quantum-proof as a completely bounded norm}

Recall that the relevant norm for extractors is a maximum of two norms denoted $\cap\left\{2^{k} \ell_{\infty}, \ell_{1}\right\}$. Our objective is to extend this norm to matrices so that it gives an operator space structure and so that the requirement of quantum-proof extractors is captured. We discussed operator space structure for the $\ell_{\infty}$ norm as well as for the $\ell_{1}$ norm. Moreover, 
it is simple to define an operator space structure for the maximum of two norms, also called intersection norm because the corresponding unit ball is the intersection of the two unit balls of the two norms. However, it turns out that because of positivity issues, this norm is not the most adapted for our purpose. In order to introduce the norm we consider, it is useful to first see the norm defined above in terms of factorization norms.

Definition V.1 (Intersection norms for extractors). We define the two operator space structures $\cap\left\{K M_{N}, S_{N}^{1}\right\}$ and $\cap \cdot \cap\left\{K M_{N}, S_{N}^{1}\right\}$ on $\operatorname{Mat}_{N}$. For $x \in \operatorname{Mat}_{Q}\left(\operatorname{Mat}_{N}\right)$, we define

$$
\|x\|_{M_{Q}\left(\cap\left\{K M_{N}, S_{N}^{1}\right\}\right)}:=\max \left\{K\|x\|_{(\infty ; \infty)},\|x\|_{(\infty ; 1)}\right\},
$$

and

$$
\|x\|_{M_{Q}\left(\cap \cdot \cap\left\{K M_{N}, S_{N}^{1}\right\}\right)}:=\inf \left\{\max \left\{\sqrt{K}\|a\|_{(\infty ; \infty)},\|\Gamma(a)\|_{\infty}\right\}, \max \left\{\sqrt{K}\|b\|_{(\infty ; \infty)},\|\Gamma(b)\|_{\infty}\right\}: x=a b^{*}\right\} .
$$

where $\Gamma \in \operatorname{Lin}\left(\operatorname{Mat}_{Q N}, \operatorname{Mat}_{Q}\left(N^{2}\right)\right)$ is defined as

$$
\Gamma(|i\rangle\langle j|\otimes| k\rangle\langle\ell|)=|i\rangle\langle j| \otimes\langle k| \otimes\langle\ell| .
$$

It might appear that the norm $\cap \cdot \cap$ is rather artificial but it can in fact be constructed from basic operator spaces (the row and column operators spaces) and a fundamental operator space tensor product called the Haagerup tensor product. For details on this we refer to Appendix B. As we always consider the intersection norms for $K=2^{k}$ times the $\infty$-norm and the 1-norm, we use the shorthand

$$
\cap:=\cap\left\{2^{k} M_{N}, S_{N}^{1}\right\} \quad \text { and } \quad \cap \cdot \cap:=\cap \cdot \cap\left\{2^{k} M_{N}, S_{N}^{1}\right\} .
$$

Observe that for extractors, we only consider matrices in $\operatorname{Mat}_{Q}(N)$. In this setting, we would write the norms as

$$
\cap=\cap\left\{2^{k} \ell_{\infty}, \ell_{1}\right\} \quad \text { and } \quad \cap \cdot \cap=\cap \cdot \cap\left\{2^{k} \ell_{\infty}, \ell_{1}\right\}
$$

The norms $\cap$ and $\cap \cdot \cap$ are actually closely related and this can be seen from the following lemma, where we write the $(\infty ; \infty)$ and $(\infty ; 1)$ norms as an infimum over all possible factorizations.

Lemma V.2. Let $x \in \operatorname{Mat}_{Q N}$. Then, we have

$$
\|x\|_{(\infty ; \infty)}=\inf \left\{\|a\|_{(\infty ; \infty)}\|b\|_{(\infty ; \infty)}: x=a b^{*} \text { and } a, b \in \operatorname{Mat}_{Q N}\right\} .
$$

as well as

$$
\|x\|_{(\infty ; 1)}=\inf \left\{\|\Gamma(a)\|_{\infty}\|\Gamma(b)\|_{\infty}: x=a b^{*} \text { and } a, b \in \operatorname{Mat}_{Q N}\right\},
$$

where $\Gamma \in \operatorname{Lin}\left(\operatorname{Mat}_{Q N}, \operatorname{Mat}_{Q}\left(N^{2}\right)\right)$ as defined in (82).

Note that in contrast, the factorization in the $\cap \cdot \cap$-norm is restricted to be the same one for the two norms.

Proof. For one direction in (85), we have for any $x=a b^{*}$ that

$$
\|x\|_{(\infty ; \infty)} \leq\|a\|_{(\infty ; \infty)}\left\|b^{*}\right\|_{(\infty ; \infty)} .
$$

For the other direction, we write a polar decomposition of $x=U P$ with $U$ unitary and $P$ positive semidefinite. Then let $a=U \sqrt{P}, b=\sqrt{P}$, and hence $\|a\|_{(\infty ; \infty)}=\|b\|_{(\infty ; \infty)}=\|P\|_{(\infty ; \infty)}^{1 / 2}$ which gives $\|a\|_{(\infty ; \infty)}\|b\|_{(\infty ; \infty)}=\|x\|_{(\infty ; \infty)}$.

For (86), we use the definition of the $(\infty ; 1)$-norm in terms of the operator space dual of $M_{N}$. For that we see $x$ as the Choi matrix of $u \in \operatorname{Lin}\left(\operatorname{Mat}_{N}, \operatorname{Mat}_{Q}\right)$, defined by

$$
\operatorname{Tr}[d u(c)]=\operatorname{Tr}\left[x\left(d \otimes c^{T}\right)\right] .
$$

We then have $\|x\|_{(\infty ; 1)}=\|u\|_{\mathrm{cb}, \infty \rightarrow \infty}$. Next we show the following useful claim: $x=a b^{*}$ is equivalent to $u(z)=$ $\hat{a}\left(z \otimes \mathbb{1}_{N Q}\right) \hat{b}^{*}$ for all $z \in \operatorname{Mat}_{N}$, where $\hat{a}=\Gamma(a)$ and $\hat{b}=\Gamma(b)$. For this, let us write $x=\sum_{i i^{\prime} k k^{\prime}} x_{i i^{\prime} k k^{\prime}}|i\rangle\left\langle i^{\prime}|\otimes| k\right\rangle\left\langle k^{\prime}\right|$. Then $x=a b^{*}$ translates to $x_{i i^{\prime} p q}=\sum_{j \ell} a_{i j p \ell} b_{i^{\prime} j q \ell}^{*}$ for all $i i^{\prime} p q$. On the other hand, we have that $\operatorname{Tr}\left[\left|i^{\prime}\right\rangle\langle i| u(|p\rangle\langle q|)\right]=$ $\operatorname{Tr}\left[x\left|i^{\prime}\right\rangle\langle i|\otimes| q\rangle\langle p|\right]=x_{i i^{\prime} p q}$. We explicitly write:

$$
\hat{a}=\sum_{i j k \ell} a_{i j k \ell}|i\rangle\langle j| \otimes\left\langle k\left|\otimes\left\langle\ell\left|\quad \hat{b}^{*}=\sum_{i j k \ell} b_{i j k \ell}^{*}\right| j\right\rangle\langle i|\otimes| k\rangle \otimes\right| \ell\right\rangle
$$


As a result,

$$
\hat{a}|p\rangle\left\langle q\left|\otimes \mathbb{1}_{N Q} \hat{b}^{*}=\sum_{i i^{\prime} j \ell} a_{i j p \ell} b_{i^{\prime} j q \ell}^{*}\right| i\right\rangle\left\langle i^{\prime}\right|,
$$

which proves the claim. To finish the proof for (86), we use [45, Theorem 5] which states that ${ }^{9}$

$$
\|u\|_{\mathrm{cb}, \infty \rightarrow \infty}=\inf \left\{\|\hat{a}\|_{\infty}\|\hat{b}\|_{\infty}: u(\beta)=\hat{a} \beta \otimes \mathbb{1}_{N Q} \hat{b}^{*}\right\} .
$$

In [45], things are written in the dual form: the diamond norm of $u^{*}$ is considered and $\hat{a}^{*}$ and $\hat{b}^{*}$ is a Stinespring pair for the channel $u^{*}$ in the sense that $u^{*}(\beta)=\operatorname{Tr}_{N Q}\left[\hat{a}^{*} \beta \hat{b}\right]$. Another point is that we can assume that the output dimension of $\hat{a}$ and $\hat{b}$ are $N Q^{2}$. This proves equality (86).

We now provide a simple bound on the $\cap \cdot \cap$-norm.

Proposition V.3. Let $x \in \operatorname{Mat}_{Q}\left(\operatorname{Mat}_{N}\right)$. Then, we have

$$
\|x\|_{M_{Q}(\cap \cdot \cap)} \geq\|x\|_{M_{Q}(\cap)},
$$

and if $x \geq 0$, we have

$$
\|x\|_{M_{Q}(\cap \cdot \cap)}=\|x\|_{M_{Q}(\cap)} .
$$

Moreover, for $Q=1$, i.e., $x \in$ Mat $_{N}$, we have $\|x\|_{\cap \cdot \cap}=\|x\|_{\cap}$.

Proof. The inequality (92) follows directly from Definition V.1 and Lemma V.2. When $x \geq 0$, the corresponding map $u \in \operatorname{Lin}\left(\operatorname{Mat}_{N}, \operatorname{Mat}_{Q}\right)$ is completely positive. This implies that the completely bounded norm of $u$, as defined in (88) is given by $\|u\|_{\mathrm{cb}, \infty \rightarrow \infty}=\|u(\mathbb{1})\|_{\infty}$. We also know that for completely positive map, we can find a representation $u(\beta)=\hat{a} \beta \otimes \mathbb{1}_{N Q} \hat{a}^{*}$. This implies that $\|x\|_{(\infty ; 1)}=\|u\|_{\mathrm{cb}, \infty \rightarrow \infty}=\left\|\hat{a} \hat{a}^{*}\right\|_{\infty}$, and then we get

$$
\|x\|_{\cap \cdot \cap} \leq\left(\max \left\{\sqrt{2^{k}}\|a\|_{\infty},\|\hat{a}\|_{\infty}\right\}\right)^{2}=\max \left\{2^{k}\|x\|_{\infty},\|x\|_{(1 ; \infty)}\right\} .
$$

For $x \in$ Mat $_{N}$, we perform a polar decomposition of $x, x=U P$ where $U$ is a unitary matrix and $P$ is positive semidefinite. Then let $a=U \sqrt{P}, b=\sqrt{P}$, and hence $\|a\|_{(\infty ; \infty)}=\|b\|_{(\infty ; \infty)}=\|P\|_{(\infty ; \infty)}^{1 / 2}$. Moreover, we have $\|\Gamma(a)\|_{\infty}=\|a\|_{2}=\|b\|_{2}=\|\sqrt{P}\|_{2}=\|P\|_{1}$, and we finally get $\max \left\{2^{k}\|x\|_{\infty},\|x\|_{1}\right\} \leq\|x\|_{\cap \cdot \cap \text {. }}$.

Proposition V.4. Let $x \in \operatorname{Mat}_{Q}\left(\operatorname{Mat}_{N}\right)$. Then, we have

$$
x=x_{1}-x_{2}+i x_{3}-i x_{4} \quad \text { with } \quad x_{i} \geq 0 \quad \text { and } \quad\left\|x_{i}\right\|_{M_{Q}(\cap \cdot \cap)} \leq\|x\|_{M_{Q}(\cap \cdot \cap)} .
$$

Proof. Let $x=a b^{*}$ be a factorization achieving the minimum of (81) with

$$
\max \left\{\sqrt{2^{k}}\|a\|_{(\infty ; \infty)},\|\Gamma(a)\|_{\infty}\right\}=\max \left\{\sqrt{2^{k}}\|b\|_{(\infty ; \infty)},\|\Gamma(b)\|_{\infty}\right\},
$$

which can be achieved by scaling $a$ and $b$. We define $x_{1}=\frac{1}{4}(a+b)(a+b)^{*}, x_{2}=\frac{1}{4}(a-b)(a-b)^{*}, x_{3}=\frac{1}{4}(a+i b)(a+i b)^{*}$ and $x_{4}=\frac{1}{4}(a-i b)(a-i b)^{*}$. It is simple to verify that $x=x_{1}-x_{2}+i x_{3}-i x_{4}$. For the norms, we have

$$
\begin{aligned}
\left\|x_{1}\right\|_{M_{Q}(\cap \cdot \cap)} & \leq \frac{1}{4} \max \left\{\sqrt{2^{k}}\|a+b\|_{(\infty ; \infty)},\|\Gamma(a+b)\|_{\infty}\right\}^{2} \\
& \leq \frac{1}{4} \max \left\{\sqrt{2^{k}}\left(\|a\|_{(\infty ; \infty)}+\|b\|_{(\infty ; \infty)}\right),\|\Gamma(a)\|_{\infty}+\|\Gamma(b)\|_{\infty}\right\}^{2} \\
& \leq \frac{1}{4}\left(\max \left\{\sqrt{2^{k}}\|a\|_{(\infty ; \infty)},\|\Gamma(a)\|_{\infty}\right\}+\max \left\{\sqrt{2^{k}}\|b\|_{(\infty ; \infty)},\|\Gamma(b)\|_{\infty}\right\}\right)^{2} \\
& =\|x\|_{M_{Q}(\cap \cdot \cap)} .
\end{aligned}
$$

The same argument also holds for $x_{i}$ with $i \in\{2,3,4\}$.

\footnotetext{
${ }^{9}$ We refer to Watrous' paper as the notation and proof are quantum information friendly, but such a result is well known in operator space theory and holds in more generality, see e.g., [28, Theorem 1.6].
} 
We now have all the operator spaces at hand that are relevant for extractors: the operator space $\cap \cdot \cap\left\{2^{k} \ell_{\infty}, \ell_{1}\right\}$ as in (81) for the input conditions and the trace class operator space $S_{N}^{1}$ with $(\infty ; 1)$ as in (68) for the output condition.

Theorem V.5. Let Ext $=\left\{f_{s}\right\}_{s \in D}$ and $\Delta[$ Ext $]$ as defined in (17). Then, we have

$$
\| \Delta[\text { Ext }]: \cap \cdot \cap\left\{2^{k} \ell_{\infty}, \ell_{1}\right\} \rightarrow \ell_{1} \|_{\mathrm{cb}} \leq \varepsilon \quad \Rightarrow \quad \text { Ext is a quantum-proof }(k, 2 \varepsilon) \text { - extractor }
$$

Ext is a quantum-proof $(k-1, \varepsilon)$-extractor $\Rightarrow \| \Delta[$ Ext $]: \cap \cdot \cap\left\{2^{k} \ell_{\infty}, \ell_{1}\right\} \rightarrow \ell_{1} \|_{\mathrm{cb}} \leq 8 \varepsilon$.

Proof. We first prove (24). For $\rho_{Q N} \in \mathcal{S}(Q N)$ with $H_{\min }(N \mid Q)_{\rho} \geq k$ we have $\left\|\rho_{Q N}\right\|_{(1 ; \infty)} \leq 2^{-k}$ as well as $\left\|\rho_{Q N}\right\|_{(1 ; 1)} \leq 1$. Hence, we get for $\sigma_{Q} \in \mathcal{S}(Q)$ that

$$
\begin{aligned}
\left\|\Delta[\operatorname{Ext}]\left(\rho_{Q N}\right)\right\|_{(1 ; 1)} & =\left\|\sigma_{Q}^{1 / 2} \Delta[\operatorname{Ext}]\left(\sigma_{Q}^{-1 / 2} \rho_{Q N} \sigma_{Q}^{-1 / 2}\right) \sigma_{Q}^{1 / 2}\right\|_{(1 ; 1)} \\
& \leq\left\|\Delta[\operatorname{Ext}]\left(\sigma_{Q}^{-1 / 2} \rho_{Q N} \sigma_{Q}^{-1 / 2}\right)\right\|_{(\infty ; 1)} \\
& \leq\|\Delta[\operatorname{Ext}]\|_{\mathrm{cb}}\left\|\sigma_{Q}^{-1 / 2} \rho_{Q N} \sigma_{Q}^{-1 / 2}\right\|_{\cap \cdot \cap} \\
& =\|\Delta[\operatorname{Ext}]\|_{\mathrm{cb}} \cdot \max \left\{2^{k}\left\|\sigma_{Q}^{-1 / 2} \rho_{Q N} \sigma_{Q}^{-1 / 2}\right\|_{(\infty ; \infty)},\left\|\sigma_{Q}^{-1 / 2} \rho_{Q N} \sigma_{Q}^{-1 / 2}\right\|_{(\infty ; 1)}\right\},
\end{aligned}
$$

where we used the simple expression for the $\cap \cdot \cap$-norm of positive operators (Proposition V.3). We now apply the previous inequality to

$$
\sigma_{Q}:=\frac{\omega_{Q}+\rho_{Q}}{2} \quad \text { with } \quad \rho_{Q N} \leq 2^{-k} \omega_{Q} \otimes \mathbb{1}_{N}
$$

and $\omega_{Q} \in \mathcal{S}(Q)$. Then, we have $2^{k} \rho_{Q N} \leq \omega_{Q} \otimes \mathbb{1}_{N} \leq 2 \sigma_{Q} \otimes \mathbb{1}_{N}$, which means

$$
2^{k}\left\|\sigma_{Q}^{-1 / 2} \rho_{Q N} \sigma_{Q}^{-1 / 2}\right\|_{(\infty ; \infty)} \leq 2
$$

In addition, as $\rho_{Q} \leq 2 \sigma$ we get

$$
\left\|\sigma_{Q}^{-1 / 2} \rho_{Q N} \sigma_{Q}^{-1 / 2}\right\|_{(\infty ; 1)}=\left\|\sigma_{Q}^{-1 / 2} \rho_{Q} \sigma_{Q}^{-1 / 2}\right\|_{\infty} \leq 2
$$

Taken (101)-(107) together proves (24).

We now prove (25). Let $z \in \operatorname{Mat}_{Q}(N)$ with $\|z\|_{\cap \cdot \cap} \leq 1$. By definition of the $\cap \cdot \cap$-norm (Definition V.1), there exists a factorization $z=a b^{*}$ such that

$$
\max \left\{\sqrt{2^{k}}\|a\|_{(\infty ; \infty)},\|\Gamma(a)\|_{\infty}\right\}=\max \left\{\sqrt{2^{k}}\|b\|_{(\infty ; \infty)},\|\Gamma(b)\|_{\infty}\right\} \leq 1 .
$$

We then define $\hat{z} \in \operatorname{Mat}_{2 Q N}$ as

$$
\hat{z}:=\left(\begin{array}{cc}
a a^{*} & b a^{*} \\
a b^{*} & b b^{*}
\end{array}\right)=(a \otimes|0\rangle\langle 0|+b \otimes| 1\rangle\langle 0|) \cdot(a \otimes|0\rangle\langle 0|+b \otimes| 1\rangle\langle 0|)^{*}
$$

and estimate

$$
\begin{aligned}
\|\hat{z}\|_{\cap \cdot \cap} & \leq\left(\max \left\{\sqrt{2^{k}} \| a \otimes|0\rangle\langle 0|+b \otimes| 0\rangle\left\langle 1\left|\left\|_{(\infty ; \infty)},\right\| \Gamma(a) \otimes\right| 0\right\rangle\langle 0|+\Gamma(b) \otimes| 0\rangle\langle 1| \|_{\infty}\right\}\right)^{2} \\
& \leq\left(2 \max \left\{\sqrt{2^{k}}\|a\|_{(\infty ; \infty)},\|\Gamma(a)\|_{\infty}\right\}\right)^{2} \\
& =4\|z\|_{\cap \cdot \cap} \\
& \leq 4
\end{aligned}
$$

By (109) we have $\hat{z} \geq 0$ and Proposition V.3 then implies

$$
\max \left\{2^{k}\|\hat{z}\|_{(\infty ; \infty)},\|\hat{z}\|_{(\infty ; 1)}\right\}=\|\hat{z}\|_{\cap \cdot \cap} \leq 4
$$


Now, we evaluate

$$
\begin{aligned}
& \|\Delta[\operatorname{Ext}](z)\|_{(\infty ; 1)}=\sup _{\substack{c, d \in \operatorname{Mat}_{Q} \\
\|c\|_{2} \leq 1,\|d\|_{2} \leq 1}}\|c \Delta[\operatorname{Ext}](z) d\|_{(1 ; 1)}=\sup _{U, c, d}|\operatorname{Tr}[c \Delta[\operatorname{Ext}](z) d \cdot U]| \\
& =\sup _{U, c, d}\left|\operatorname{Tr}\left[\Delta[\operatorname{Ext}]\left(\left(\begin{array}{ll}
c & 0 \\
0 & c
\end{array}\right) \hat{z}\left(\begin{array}{ll}
d & 0 \\
0 & d
\end{array}\right)\right) \cdot U \otimes|1\rangle\langle 0|\right]\right| .
\end{aligned}
$$

For the positive semidefinite operator

$$
\rho:=\frac{1}{8}\left(\begin{array}{ll}
c & 0 \\
0 & c
\end{array}\right) \hat{z}\left(\begin{array}{ll}
d & 0 \\
0 & d
\end{array}\right)
$$

we get by the definition of the $(1 ; \infty)$-norm in $(50)$ as well as $(114)$ that

$$
\|\rho\|_{(1 ; \infty)} \leq \frac{1}{8} \cdot 2\|\hat{z}\|_{(\infty ; \infty)} \leq 2^{-k} \text { and }\|\rho\|_{(1 ; 1)} \leq \frac{1}{8} \cdot 2\|\hat{z}\|_{(\infty ; 1)} \leq 1 .
$$

In order to have a valid state, we define

$$
\bar{\rho}:=\rho+(1-\operatorname{Tr}[\rho]) \frac{\mathbb{1}}{Q N} \quad \text { with } \quad\|\bar{\rho}\|_{(1 ; \infty)} \leq 2^{-k}+\frac{1}{N} \leq 2^{-(k-1)} .
$$

Now, by assumption Ext is a quantum-proof $(k-1, \varepsilon)$-extractor and with (115)-(116) we conclude that

$$
\|\Delta[\operatorname{Ext}](z)\|_{(\infty ; 1)} \leq 8 \cdot\|\Delta[\operatorname{Ext}](\rho)\|_{(1 ; 1)} \leq 8 \cdot\|\Delta[\operatorname{Ext}](\bar{\rho})\|_{(1 ; 1)} \leq 8 \varepsilon .
$$

\section{Stability bounds}

This way of writing the extractor and the quantum-proof extractor conditions allows us to use tools from operator space theory to compare the two concepts. As a first straightforward application, we can use dimension dependent bounds that are known for the maximal possible ratios between the completely bounded norm and the bounded norm.

Corollary V.6. Every $(k, \varepsilon)$-extractor is a quantum-proof $\left(k+1,6 \sqrt{2^{m}} \varepsilon\right)$-extractor.

Observe that this result is only interesting when $m$ is small. In particular, it is only useful for weak extractors, as strong extractors have $2^{m} \geq 2^{d}=\Omega\left(\varepsilon^{-2}\right)$ (by the converse bound (4)).

Proof. By Proposition II.1 we have that $\| \Delta\left[\right.$ Ext] $: \cap \cap\left\{2^{k+1} \ell_{\infty}, \ell_{1}\right\} \rightarrow \ell_{1} \| \leq 3 \varepsilon$. Now, we estimate using [28, Theorem $3.8]$,

$$
\begin{aligned}
\| \Delta[\text { Ext }]: \cap \cdot \cap\left\{2^{k+1} \ell_{\infty}, \ell_{1}\right\} \rightarrow \ell_{1} \|_{\mathrm{cb}} & \leq \| \Delta[\text { Ext }]: \cap \cdot \cap\left\{2^{k+1} \ell_{\infty}, \ell_{1}\right\} \rightarrow \min \left(\ell_{1}\right)\left\|_{\mathrm{cb}} \cdot\right\| \mathbb{1}: \min \left(\ell_{1}\right) \rightarrow \ell_{1} \|_{\mathrm{cb}} \\
& =\| \Delta\left[\text { Ext] }: \cap \cdot \cap\left\{2^{k+1} \ell_{\infty}, \ell_{1}\right\} \rightarrow \ell_{1}\|\cdot\| \mathbb{1}: \ell_{\infty} \rightarrow \max \left(\ell_{\infty}\right) \|_{\mathrm{cb}}\right. \\
& \leq 3 \varepsilon \sqrt{2^{m}} .
\end{aligned}
$$

The claim then follows by Theorem II.2.

Theorem V.7. Let Ext $=\left\{f_{s}\right\}_{s \in D}$, and $\Delta[\text { Ext }]_{S}$ as defined in (22). Then, we have

$$
\left\|\Delta[\mathrm{Ext}]_{S}: \cap\left\{2^{k} \ell_{\infty}, \ell_{1}\right\} \rightarrow \ell_{1}\right\| \leq \varepsilon \Rightarrow\left\|\Delta[\mathrm{Ext}]_{S}: \cap \cdot \cap\left\{2^{k+\log (1 / \varepsilon)} \ell_{\infty}, \ell_{1}\right\} \rightarrow \ell_{1}\right\|_{\mathrm{cb}} \leq 4 \sqrt{2^{m^{\prime}}} \sqrt{2 \varepsilon} .
$$

Proof. By operator space duality for the trace class operator space (Proposition IV.3) and Proposition V.4 we get

$$
\left\|\Delta[\mathrm{Ext}]_{S}: \cap \cdot \cap\left\{2^{k+\log (1 / \varepsilon)} \ell_{\infty}, \ell_{1}\right\} \rightarrow \ell_{1}\right\|_{\mathrm{cb}} \leq 4 \sup \left\{\left\|\mathbb{E}_{s} \sum_{y} \sum_{x}\left(\delta_{f_{s}(x)=y}-2^{-m}\right) p(x) \otimes q(s, y)\right\|_{(\infty ; \infty)}\right\}
$$


where the supremum is over all

$$
\begin{aligned}
p(x) \in \operatorname{Mat}_{Q} \quad \text { with } & 0 \leq p(x) \leq \varepsilon 2^{-k} \mathbb{1}, \sum_{x} p(x) \leq \mathbb{1} \\
q(s, y) \in \operatorname{Mat}_{Q} \quad \text { with } & \|q(s, y)\|_{\infty} \leq 1
\end{aligned}
$$

and $Q \in \mathbb{N}$. We now apply the operator version of the Cauchy-Schwarz inequality due to Haagerup (see, e.g., [28, Chapter 7]),

$$
\begin{aligned}
& \left\|\mathbb{E}_{s} \sum_{y}\left(\sum_{x}\left(\delta_{f_{s}(x)=y}-2^{-m^{\prime}}\right) p(x)\right) \otimes q(s, y)\right\|_{(\infty ; \infty)} \\
& \leq\left\|\mathbb{E}_{s} \sum_{y}\left(\sum_{x}\left(\delta_{f_{s}(x)=y}-2^{-m^{\prime}}\right) p(x)\right) \otimes \overline{\left(\sum_{x^{\prime}}\left(\delta_{f_{s}\left(x^{\prime}\right)=y}-2^{-m^{\prime}}\right) p\left(x^{\prime}\right)\right)}\right\|_{(\infty ; \infty)}^{1 / 2}\left\|\mathbb{E}_{s} \sum_{y} q(s, y) \otimes \overline{q(s, y)}\right\|_{(\infty ; \infty)}^{1 / 2} .
\end{aligned}
$$

The second term is upper bounded by

$$
\left\|\mathbb{E}_{s} \sum_{y} q(s, y) \otimes \overline{q(s, y)}\right\|_{(\infty ; \infty)} \leq \mathbb{E}_{s} \sum_{y}\|q(s, y)\|_{\infty}^{2} \leq \sqrt{2^{m^{\prime}}}
$$

and we are left with the first term. The operator whose $(\infty ; \infty)$-norm has to be estimated is hermitian, and hence we arrive via norm duality at

$$
\begin{aligned}
& \sup _{\substack{C \in \text { Mat } \\
\operatorname{Tr}\left[C^{*} C\right] \leq 1}}\left|\mathbb{E}_{s} \sum_{y} \sum_{x, x^{\prime}}\left(\delta_{f_{s}(x)=y}-2^{-m^{\prime}}\right) \operatorname{Tr}\left[C p(x) C^{*} p\left(x^{\prime}\right)\right]\left(\delta_{f_{s}\left(x^{\prime}\right)=y}-2^{-m^{\prime}}\right)\right| \\
& \leq \sup _{\substack{C \in \text { Mat } \\
\operatorname{Tr}\left[C^{*} C\right] \leq 1}} \mathbb{E}_{s} \sum_{y} \sum_{x^{\prime}}\left|\sum_{x}\left(\delta_{f_{s}(x)=y}-2^{-m^{\prime}}\right) l_{x x^{\prime}}(C)\right|
\end{aligned}
$$

where $l_{x x^{\prime}}(C):=\operatorname{Tr}\left[C p(x) C^{*} p\left(x^{\prime}\right)\right]$ is a positive function on $N \times N$ with

$$
\sum_{x, x^{\prime}} l_{x x^{\prime}}(C)=\operatorname{Tr}\left[C \sum_{x} p(x) C^{*} \sum_{x^{\prime}} p\left(x^{\prime}\right)\right] \leq 1 \quad \text { and } \quad l_{x x^{\prime}}(C) \leq \varepsilon 2^{-k} \operatorname{Tr}\left[C C^{*} p\left(x^{\prime}\right)\right]
$$

Hence, the distribution $l_{x x^{\prime}}(C)$ has conditional min-entropy $H_{\min }\left(X \mid X^{\prime}\right)_{l(C)} \geq k+\log (1 / \varepsilon)$, and by Markov's inequality

$$
\mathbf{P}\left\{H_{\min }\left(X \mid X^{\prime}=x^{\prime}\right)_{l(C)} \leq k\right\} \leq \varepsilon
$$

Finally, we get by the assumption that the bounded norm of the extractor is upper bounded by $\varepsilon$ that

$$
\begin{aligned}
\mathbb{E}_{s} \sum_{y} \sum_{x^{\prime}}\left|\sum_{x}\left(\delta_{f_{s}(x)=y}-2^{-m^{\prime}}\right) l_{x x^{\prime}}(C)\right| & =\mathbb{E}_{x^{\prime}} \mathbb{E}_{s} \sum_{y}\left|\sum_{x}\left(\delta_{f_{s}(x)=y}-2^{-m^{\prime}}\right) l_{x \mid x^{\prime}}(C)\right| \\
& \leq 2 \varepsilon .
\end{aligned}
$$

By putting everything together we get the upper bound $4 \sqrt{2^{m^{\prime}}} \sqrt{2 \varepsilon}$ on the completely bounded norm of the extractor as claimed.

An interesting application is for very high min-entropy extractors.

Theorem V.8. Let Ext $=\left\{f_{s}\right\}_{s \in D}$ and $\Delta[$ Ext $]$ as defined in (17). Then, we have

$$
\| \Delta[\text { Ext }]: \cap\left\{2^{k} \ell_{\infty}, \ell_{1}\right\} \rightarrow \ell_{1}\|\leq \varepsilon \Rightarrow\| \Delta[\text { Ext }]: \cap \cdot \cap\left\{2^{k} \ell_{\infty}, \ell_{1}\right\} \rightarrow \ell_{1} \|_{\mathrm{cb}} \leq K_{G} 2^{n-k} \varepsilon .
$$


Proof. We have for any distribution $P$,

$$
\|P\|_{2^{k} \ell_{\infty}} \leq\|P\|_{\cap\left(2^{k} \ell_{\infty}, \ell_{1}\right)} \leq 2^{n-k}\|P\|_{2^{k} \ell_{\infty}},
$$

and this implies that

$$
\left\|\Delta[\mathrm{Ext}]: 2^{k+1} \ell_{\infty} \rightarrow \ell_{1}\right\| \leq 2^{n-k} \varepsilon .
$$

By Grothendieck's inequality [29, Corollary 14.2], we conclude

$$
\| \Delta[\text { Ext }]: \cap \cdot \cap\left\{2^{k} \ell_{\infty}, \ell_{1}\right\} \rightarrow \ell_{1}\left\|_{\mathrm{cb}} \leq\right\| \Delta[\mathrm{Ext}]: 2^{k} \ell_{\infty} \rightarrow \ell_{1} \|_{\mathrm{cb}} \leq K_{G} 2^{n-k} \varepsilon .
$$

\section{CONDENSERS}

\section{A. Condenser property as a bounded norm}

Proposition VI.1. Let $\mathrm{Con}=\left\{f_{s}\right\}_{s \in D}$ and $[\mathrm{Con}]$ as defined in (30). Then, we have

$$
\begin{array}{ccc}
\|[\text { Con }]: \cap\left\{2^{k} \ell_{\infty}, \ell_{1}\right\} \rightarrow \Sigma\left\{2^{k^{\prime}} \ell_{\infty}, \ell_{1}\right\} \| \leq \varepsilon & \Rightarrow \text { Con is a }\left(k \rightarrow_{\varepsilon} k^{\prime}+\log (1 / \varepsilon)\right) \text { - condenser } \\
\text { Con is a }\left(k-1 \rightarrow_{\varepsilon} k^{\prime}+\log (1 / \varepsilon)\right) \text { - condenser } & \Rightarrow \quad \|[\text { Con }]: \cap\left\{2^{k} \ell_{\infty}, \ell_{1}\right\} \rightarrow \Sigma\left\{2^{k^{\prime}} \ell_{\infty}, \ell_{1}\right\} \| \leq 6 \varepsilon .
\end{array}
$$

Proof. We first prove (33). Let $P_{N}$ be a distribution with min-entropy at least $k$. Then, we have $\|P\|_{\cap\left(2^{k} \ell_{\infty}, \ell_{1}\right)} \leq 1$ and this implies $\|[\mathrm{Con}](P)\|_{\Sigma\left\{2^{k^{\prime}} \ell_{\infty}, \ell_{1}\right\}} \leq \varepsilon$. Hence, there is a decomposition $[\mathrm{Con}](P)=Q_{1}+Q_{2}$ such that $\left\|Q_{1}\right\|_{\ell_{\infty}} \leq \varepsilon 2^{-k}$ and $\left\|Q_{2}\right\|_{\ell_{1}}=\left\|P-Q_{1}\right\|_{\ell_{1}} \leq \varepsilon$. In order to conclude that $H_{\min }^{\varepsilon}(P) \geq k^{\prime}+\log (1 / \varepsilon)$, it only remains to show that $Q_{1}$ and $Q_{2}$ are nonnegative. In fact, consider $Q_{1}^{\prime}=\max \left(Q_{1}, 0\right)$ and $Q_{2}^{\prime}=P-Q_{1}^{\prime}=\min \left(Q_{2}, P\right)$. Then, we still have $Q_{1}^{\prime}+Q_{2}^{\prime}=P$, and in addition $\left\|Q_{1}^{\prime}\right\|_{2^{k^{\prime}} \ell_{\infty}} \leq\left\|Q_{1}\right\|_{2^{k^{\prime}} \ell_{\infty}}$ as well as $\left\|Q_{2}^{\prime}\right\|_{1} \leq\left\|Q_{2}\right\|_{\ell_{1}}$. This proves that without lost of generality $Q_{1}$ and $Q_{2}$ are nonnegative.

To prove (34), assume that Con defines a $(k-1) \rightarrow_{\varepsilon} k^{\prime}+\log (1 / \varepsilon)$ and consider $P$ such that $\|P\|_{\ell_{1}} \leq 1$ and $\|P\|_{\ell_{\infty}} \leq 2^{-k}$. Let $P^{+}(x)=\max \{P(x), 0\}$ and $P^{-}(x)=\max \{-P(x), 0\}$, and note that $\left\|P^{+}\right\|_{\ell_{\infty}},\left\|P^{-}\right\|_{\ell_{\infty}} \leq 2^{-k}$. We extend $P^{+}$into a probability distribution $\bar{P}^{+}=P^{+}+\left(1-\left\|P^{+}\right\|_{\ell_{1}}\right) v_{N}$ and similarly $\bar{P}^{-}(x)=P^{-}(x)+\left(1-\left\|P^{-}\right\|_{\ell_{1}}\right) v_{N}$. Now observe that $\left\|\bar{P}^{+}\right\|_{\ell_{\infty}} \leq\|P\|_{\ell_{\infty}}+\frac{1}{N} \leq 2^{-(k-1)}$ and similarly for $\left\|\bar{P}^{+}\right\|_{\ell_{\infty}}$. Thus, we have that

$$
\begin{aligned}
\|[\operatorname{Con}](P)\|_{\ell_{1}}= & \left\|[\operatorname{Con}]\left(P^{+}\right)-[\operatorname{Con}]\left(P^{-}\right)\right\|_{\Sigma\left\{2^{k^{\prime}} \ell_{\infty}, \ell_{1}\right\}} \\
\leq & \left\|[\operatorname{Con}]\left(P^{+}\right)\right\|_{\Sigma\left\{2^{k^{\prime}} \ell_{\infty}, \ell_{1}\right\}}+\left\|[\operatorname{Con}]\left(P^{-}\right)\right\|_{\Sigma\left\{2^{k^{\prime}} \ell_{\infty}, \ell_{1}\right\}} \\
\leq & \left\|[\operatorname{Con}]\left(\bar{P}^{+}\right)\right\|_{\Sigma\left\{2^{k^{\prime}} \ell_{\infty}, \ell_{1}\right\}}+\left(1-\left\|P^{+}\right\|_{\ell_{1}}\right)\left\|[\operatorname{Con}]\left(v_{N}\right)\right\|_{\Sigma\left\{2^{k^{\prime}} \ell_{\infty}, \ell_{1}\right\}} \\
& +\left\|[\operatorname{Con}]\left(\bar{P}^{-}\right)\right\|_{\Sigma\left\{2^{k^{\prime}} \ell_{\infty}, \ell_{1}\right\}}+\left(1-\left\|P^{-}\right\|_{\ell_{1}}\right)\left\|[\operatorname{Con}]\left(v_{N}\right)\right\|_{\Sigma\left\{2^{k^{\prime}} \ell_{\infty}, \ell_{1}\right\}} \\
\leq & \left\|[\operatorname{Con}]\left(\bar{P}^{+}\right)\right\|_{\Sigma\left\{2^{k^{\prime}} \ell_{\infty}, \ell_{1}\right\}}+\left\|[\operatorname{Con}]\left(\bar{P}^{-}\right)\right\|_{\Sigma\left\{2^{k^{\prime}} \ell_{\infty}, \ell_{1}\right\}}+\left\|[\operatorname{Con}]\left(v_{N}\right)\right\|_{\Sigma\left\{2^{k^{\prime}} \ell_{\infty}, \ell_{1}\right\}} .
\end{aligned}
$$

Now the distributions $\bar{P}^{+}, \bar{P}^{-}$and $v_{N}$ have min-entropy at least $k-1$. Hence, there exists $Q$ with $\|P-Q\|_{\ell_{1}} \leq \varepsilon / 6$ and $\|Q\|_{2^{k^{\prime}} \ell_{\infty}} \leq \varepsilon / 6$. This then implies $\|P\|_{\Sigma\left\{2^{k^{\prime}} \ell_{\infty}, \ell_{1}\right\}} \leq \varepsilon / 3$, which proves the desired result.

\section{B. Quantum-proof as a completely bounded norm}

As we saw in Proposition II.1, a condenser maps the $\cap$-normed space to its dual space. Since we expect the same to happen in the quantum-proof case, it is useful to have an understanding about the operator space dual of $\cap \cdot \cap$. By expressing the $\cap \cdot \cap$-operator space using the Haagerup tensor product, the operator dual is easily identified. However, we do not want to elaborate further on this, since we will just use a simple estimate (see Lemma VI.2 below), and refer to Appendix B for the exact characterization. We again use a shorthand notation and denote the operator space dual of $\cap \cdot \cap$ by $\Sigma \cdot \Sigma$. In the setting of this paper, we are naturally interested in the case of matrices which are diagonal with respect to the first system, that is, elements of $\operatorname{Mat}_{Q}(N)$. The norms $\cap \cdot \cap$ and the $\Sigma \cdot \Sigma$ are then defined on $\operatorname{Mat}_{Q}(N)$ via the embedding of $\operatorname{Mat}_{Q}(N)$ into $\operatorname{Mat}_{Q N}$ as block-diagonal matrices. To emphasize this case, we again use the notation

$$
\cap \cdot \cap\left\{2^{k} \ell_{\infty}, \ell_{1}\right\} \quad \text { as well as } \Sigma \cdot \Sigma\left\{\ell_{\infty}, 2^{-k} \ell_{1}\right\}=2^{-k} \Sigma \cdot \Sigma\left\{2^{k} \ell_{\infty}, \ell_{1}\right\}
$$


for the corresponding dual space. For positive $x \in \operatorname{Mat}_{Q}(N)$, the operator space dual norm $\Sigma \cdot \Sigma$ has the following simple estimate.

Lemma VI.2. Let $x=\sum_{j} x(j) \otimes|i\rangle\langle i| \in \operatorname{Mat}_{Q}(N)$ be positive. Then, we have

$$
\frac{1}{2}\|x\|_{M_{Q}(\Sigma \cdot \Sigma)} \leq \inf \left\{\left\|2^{-k} \sum_{j} A(j)+B\right\|_{\infty}: x(j) \leq A(j)+B, A(j) \geq 0, B \geq 0\right\} \leq\|x\|_{M_{Q}(\Sigma \cdot \Sigma)} .
$$

Proof. By the definition of the operator space dual, we have

$$
\|x\|_{M_{Q}(\Sigma \cdot \Sigma)}=\sup \left\{\left\|\sum_{j} x(j) \otimes y(j)\right\|_{(\infty ; \infty)}: \| \sum_{j} y(j) \otimes|j\rangle\langle j| \|_{M_{Q}(\cap \cdot \cap)} \leq 1\right\} .
$$

Let us now decompose $y$ according to Proposition V.4, $y=y_{1}-y_{2}+i y_{3}-i y_{4}$. Then, we observe that in the maximization in (143), up to an additional factor of two we can assume that $y$ is also Hermitian. This implies that we can take $y_{3}=y_{4}=0$. But then using the fact that $x \geq 0$, we have

$$
\left\|\sum_{j} x(j) \otimes y(j)\right\|_{(\infty ; \infty)} \leq\left\|\sum_{j} x(j) \otimes y_{1}(j)\right\|_{(\infty ; \infty)}
$$

and thus we can assume that $y \geq 0$ in the further study of (143). Recalling that when $y \geq 0$,

$$
\|y\|_{M_{Q}(\cap \cdot \cap)}=\|y\|_{M_{Q}(\cap)}=\max \left\{2^{k}\|y\|_{\infty},\left\|\sum_{j} y(j)\right\|_{\infty}\right\}
$$

we have

$$
\begin{aligned}
& \sup \left\{\left\langle\psi\left|\sum_{j} x(j) \otimes y(j)\right| \psi\right\rangle: 0 \leq y(j) \leq 2^{-k} \mathbb{1}, \sum_{j} y(j) \leq \mathbb{1}, \||\psi\rangle \|_{2} \leq 1\right\} \\
& =\sup \left\{\sum_{j, l, l^{\prime}} \lambda_{l}^{*} \lambda_{l}\left\langle u_{l}|x(j)| u_{l^{\prime}}\right\rangle\left\langle v_{l}|y(j)| v_{l^{\prime}}\right\rangle: 0 \leq y(j) \leq 2^{-k} \mathbb{1}, \sum_{j} y(j) \leq \mathbb{1} \||\psi\rangle \|_{2} \leq 1\right\},
\end{aligned}
$$

where $|\psi\rangle=\sum_{l} \lambda_{l}\left|u_{l}\right\rangle\left|v_{l}\right\rangle$ is a Schmidt decomposition. Now using the fact that $\left\langle v_{l}|y(j)| v_{l^{\prime}}\right\rangle=\left\langle v_{l^{\prime}}\left|y(j)^{T}\right| v_{l}\right\rangle$ and writing $C=\sum_{l} \lambda_{l}\left|v_{l}\right\rangle\left\langle u_{l}\right|$, we can write

$$
\|x\|_{M_{Q}(\Sigma \cdot \Sigma)}=\sup \left\{\operatorname{Tr}\left[\sum_{j} x(j) C^{*} y(j)^{T} C\right]: 0 \leq y(j) \leq 2^{-k} \mathbb{1}, \sum_{j} y(j) \leq \mathbb{1}, \operatorname{Tr}\left[C^{*} C\right] \leq 1\right\}
$$

Moreover, the transposition leaves the operator inequalities involving the $y(j)$ 's invariant, we can further simplify to $\left(\operatorname{set} \sigma=C^{*} C\right)$

$$
\sup \left\{\operatorname{Tr}\left[\sum_{j} x(j) \hat{y}(j)\right]: 0 \leq \hat{y}(j) \leq 2^{-k} \sigma, \sum_{j} \hat{y}(j) \leq \sigma, \operatorname{Tr}[\sigma] \leq 1, \sigma \geq 0\right\}
$$

This is an SDP and its dual is given by

$$
\inf \left\{\left\|2^{-k} \sum_{j} A(j)+B\right\|_{\infty}: x(j) \leq A(j)+B, A(j) \geq 0, B \geq 0\right\} .
$$

The program (148) is clearly feasible and (149) is strictly feasible and thus strong duality is satisfied, i.e., the programs (148) and (149) have the same value. 
Such kind of intersection norms for operator spaces have been extensively employed by Junge and his co-workers in their study of non-commutative $L_{p}$-spaces and their relation to free probability, see for instance the monograph [20]. ${ }^{10}$ We first need a lemma relating the dual norm of $\cap \cdot \cap$ for positive elements to the smooth conditional min-entropy.

Lemma VI.3. Let $\rho \in \operatorname{Mat}_{Q N}$ be positive. Then, we have

$$
\begin{array}{rll}
2^{k}\|\rho\|_{M_{Q}(\Sigma \cdot \Sigma)} \leq \varepsilon & \Rightarrow \quad H_{\min }^{2 \sqrt{\varepsilon}}(N \mid Q)_{\rho} \geq k+\log (1 / \varepsilon) \\
H_{\min }^{\varepsilon}(N \mid Q) \geq k+\log (1 / \varepsilon) & \Rightarrow \quad 2^{k}\|\rho\|_{M_{Q}(\Sigma \cdot \Sigma)} \leq 2 \varepsilon .
\end{array}
$$

Proof. We first prove (150). Due to the fact that $\rho$ is positive, the optimal decomposition of $x$ with respect to Proposition V.4 is one where only the positive term is non-zero. Hence, we have due to Proposition V.3,

$$
\begin{aligned}
\|\rho\|_{M_{Q}(\Sigma \cdot \Sigma)}=\sup \left\{|\operatorname{Tr}[\rho x]|:\|x\|_{\cap \cdot \cap\left\{2^{k} \ell_{\infty}, \ell_{1}\right\}} \leq 1\right\} & =\sup \left\{|\operatorname{Tr}[\rho x]|: x \geq 0,\|x\|_{\cap\left\{2^{k} \ell_{\infty}, \ell_{1}\right\}} \leq 1\right\} \\
& =2^{-k} \sup \left\{\operatorname{Tr}[\rho x]: x \geq 0, \operatorname{Tr}_{N}(x) \leq 2^{k} \mathbb{1}, x \leq \mathbb{1}\right\} .
\end{aligned}
$$

This is a semidefinite program, and by strong duality we get

$$
\sup \left\{\operatorname{Tr}[\rho x]: x \geq 0, \operatorname{Tr}_{N}(x) \leq 2^{k} \mathbb{1}, x \leq \mathbb{1}\right\}=\inf \left\{\operatorname{Tr}\left[A_{1}\right]+2^{k} \operatorname{Tr}\left[A_{2}\right]: \rho \leq A_{1}+\mathbb{1}_{N} \otimes A_{2}, A_{1,2} \geq 0\right\} .
$$

Hence, we find two positive matrices $A_{1}$ and $A_{2}$ such that $\operatorname{Tr}\left[A_{1}\right] \leq \varepsilon$, and $\operatorname{Tr}\left[A_{2}\right] \leq \varepsilon 2^{-k}$. We now consider

$$
\hat{\rho}:=B^{*} B \quad \text { with } \quad B:=\rho^{1 / 2}\left(A_{1}+\mathbb{1} \otimes A_{2}\right)^{-1 / 2} \mathbb{1} \otimes A_{2}^{1 / 2} .
$$

We have that its trace is smaller than one,

$$
\begin{aligned}
\operatorname{Tr}[\hat{\rho}] & =\operatorname{Tr}\left[\mathbb{1} \otimes A_{2}^{1 / 2}\left(A_{1}+\mathbb{1} \otimes A_{2}\right)^{-1 / 2} \rho\left(A_{1}+\mathbb{1} \otimes A_{2}\right)^{-1 / 2} \mathbb{1} \otimes A_{2}^{1 / 2}\right] \\
& =\operatorname{Tr}\left[\rho\left(A_{1}+\mathbb{1} \otimes A_{2}\right)^{-1 / 2} \mathbb{1} \otimes A_{2}\left(A_{1}+\mathbb{1} \otimes A_{2}\right)^{-1 / 2}\right] \\
& \leq \operatorname{Tr}[\rho] \\
& =1,
\end{aligned}
$$

since $\left(A_{1}+\mathbb{1} \otimes A_{2}\right)^{-1 / 2} \mathbb{1} \otimes A_{2}\left(A_{1}+\mathbb{1} \otimes A_{2}\right)^{-1 / 2} \leq\left(A_{1}+\mathbb{1} \otimes A_{2}\right)^{-1 / 2} A_{1}+\mathbb{1} \otimes A_{2}\left(A_{1}+\mathbb{1} \otimes A_{2}\right)^{-1 / 2}=\mathbb{1}$. This implies that $\hat{\rho}$ is a sub-normalized state, and its min-entropy is as least $k+\log (1 / \varepsilon)$ since we have $\left(A_{1}+\mathbb{1} \otimes A_{2}\right)^{-1 / 2} \rho\left(A_{1}+\mathbb{1} \otimes A_{2}\right)^{-1 / 2} \leq$ $\mathbb{1}$ by construction and $\operatorname{Tr}\left[A_{2}\right] \leq \varepsilon 2^{-k}$. Simple rescaling of $A_{2}$ makes it into a density matrix, and the corresponding factor is picked up by the inner term. Moreover, let us consider its trace norm distance to $\rho$. First, we have using the bounded trace of $\rho$ and $B^{*} B$,

$$
\left\|\rho-B^{*} B\right\|_{1}=\left\|\left(\rho^{1 / 2}-B^{*}\right) \rho^{1 / 2}+B^{*}\left(\rho^{1 / 2}-B\right)\right\|_{1} \leq 2\left\|\rho^{1 / 2}-B\right\|_{2} .
$$

We then estimate further, using the Powers-Stoermer inequality in the last step,

$$
\begin{aligned}
\left\|\rho^{1 / 2}-B\right\|_{2} & =\left\|\rho^{1 / 2}\left(A_{1}+\mathbb{1} \otimes A_{2}\right)^{-1 / 2}\left(A_{1}+\mathbb{1} \otimes A_{2}\right)^{1 / 2}-\rho^{1 / 2}\left(A_{1}+\mathbb{1} \otimes A_{2}\right)^{-1 / 2} \mathbb{1} \otimes A_{2}^{1 / 2}\right\|_{2} \\
& \leq\left\|\rho^{1 / 2}\left(A_{1}+\mathbb{1} \otimes A_{2}\right)^{-1 / 2}\right\|_{\infty} \cdot\left\|\left(A_{1}+\mathbb{1} \otimes A_{2}\right)^{1 / 2}-\mathbb{1} \otimes A_{2}^{1 / 2}\right\|_{2} \\
& \leq \sqrt{\left\|A_{1}\right\|_{1}} \\
& =\sqrt{\varepsilon}
\end{aligned}
$$

and the first statement follows.

To prove (151), let $\hat{\rho}$ be the state which achieves the smooth min-entropy, i.e., we have $\hat{\rho} \leq \varepsilon 2^{-k} \mathbb{1} \otimes \sigma$ and $\|\hat{\rho}-\rho\|_{1} \leq \varepsilon$. Let $x$ be now chosen such that $\operatorname{Tr}_{N}(x) \leq 2^{k} \mathbb{1}, x \leq \mathbb{1}$. We then have

$$
2^{-k} \operatorname{Tr}[x \rho]=\operatorname{Tr}[x \hat{\rho}]+\operatorname{Tr}[x(\hat{\rho}-\rho)] \leq \varepsilon 2^{-k} \operatorname{Tr}\left[\sigma \operatorname{Tr}_{N}[x]\right]+\varepsilon\|x\|_{\infty} \leq 2 \cdot 2^{-k} \varepsilon,
$$

and the assertion is proven.

10 We expect that many of Junge and his co-workers' techniques are applicable to questions regarding the stability of pseudorandmom objects and hope that our work serves as a starting point for such kind of investigations. 
Expressing the smooth conditional min-entropy in terms of the $\Sigma \cdot \Sigma$-norm (Lemma VI.3) allows us to characterize quantum-proof condensers using operator space norms.

Theorem VI.4. Let Con $=\left\{f_{s}\right\}_{s \in D}$ and [Con] as defined in (30). Then, we have

$$
\|[\text { Con }]: \cap \cdot \cap\left\{2^{k} \ell_{\infty}, \ell_{1}\right\} \rightarrow \Sigma \cdot \Sigma\left\{2^{k^{\prime}} \ell_{\infty}, \ell_{1}\right\} \|_{\mathrm{cb}} \leq \frac{\varepsilon}{4} \Rightarrow \text { Con is a quantum-proof }\left(k \rightarrow_{\varepsilon} k^{\prime}+\log (1 / \varepsilon)\right)-\operatorname{condenser},
$$

Con is a quantum-proof $\left(k-1 \rightarrow_{\varepsilon} k^{\prime}+\log (1 / \varepsilon)\right)$ - condenser $\Rightarrow \quad \|[$ Con $]: \cap \cdot \cap\left\{2^{k} \ell_{\infty}, \ell_{1}\right\} \rightarrow \Sigma \cdot \Sigma\left\{2^{k^{\prime}} \ell_{\infty}, \ell_{1}\right\} \|_{\mathrm{cb}} \leq 8 \varepsilon$.

Proof. We first prove (37). Let $\rho_{N Q} \in \operatorname{Mat}_{Q}(N)$ be a state with conditional min-entropy at least $k$, and let $\omega \in$ Mat $_{Q}$ such that $\rho_{N Q} \leq 2^{-k} \omega \otimes \mathbb{1}_{N}$. We proceed as in the proof of Theorem II.2 and define $\sigma=\frac{\rho_{Q}+\omega}{2}$. It follows that $\left\|\sigma^{-1 / 2} \rho \sigma^{-1 / 2}\right\|_{\cap \cdot \cap\left[2^{k} \ell_{\infty}, \ell_{1}\right]} \leq 2$ and hence we have due to Lemma VI.2 that there are positive matrices $A \in \operatorname{Mat}_{Q}(N)$, $B \in \operatorname{Mat}_{Q}$ such that

$$
\operatorname{Con}(\rho)=\sigma^{1 / 2} \operatorname{Con}\left(\sigma^{-1 / 2} \rho \sigma^{-1 / 2}\right) \sigma^{1 / 2} \leq \sigma^{1 / 2}(A+\mathbb{1} \otimes B) \sigma^{1 / 2} \text { and }\left(\sum_{y \in M} A(y)+2^{k^{\prime}} B\right) \leq \varepsilon \mathbb{1} .
$$

Now take $x \in \operatorname{Mat}_{Q}(M)$, fulfilling $x \geq 0, x \leq \mathbb{1}$ and $\sum_{y \in M} x(y) \leq 2^{k^{\prime}} \mathbb{1}$. Then, we have

$$
\begin{aligned}
\operatorname{Tr}[\rho x] \leq \operatorname{Tr}\left[\sigma^{1 / 2}\left(A+\mathbb{1}_{M} \otimes B\right) \sigma^{1 / 2} x\right] & \leq \operatorname{Tr}_{Q}\left[\sigma^{1 / 2} \sum_{y \in M} A(y) \sigma^{1 / 2}\right]+\operatorname{Tr}_{Q}\left[\sigma^{1 / 2} B \sigma^{1 / 2} \sum_{y \in M} x(y)\right] \\
& \left.\leq \operatorname{Tr}\left[\sigma\left(\sum_{y \in M} A(y)+2^{k^{\prime}} B\right)\right]\right] \\
& \leq \varepsilon .
\end{aligned}
$$

and (37) follows by Lemma VI.3.

For proving (38), take $x \in \operatorname{Mat}_{Q}(N)$ with $\|x\|_{\cap \cdot \cap\left\{2^{k} \ell_{\infty}, \ell_{1}\right\}} \leq 1$. Due to Proposition V.4 we can find a decomposition of $x=x_{1}-x_{2}+i\left(x_{3}-x_{4}\right)$ into positive terms fulfilling the same norm constraint. We will proceed to bound

$$
\left\|\sum_{y \in M} z(y) \otimes \operatorname{Con}\left(x_{i}\right)(y)\right\|_{(\infty ; \infty)} \leq \varepsilon
$$

where $\|z\|_{\cap \cdot \cap\left\{2^{k^{\prime}} \ell_{\infty}, \ell_{1}\right\}} \leq 2^{k^{\prime}}$. This implies the assertion by operator space duality. Due to the fact that Con $\left(x_{i}\right)(y)$ is positive, the optimal decomposition of $z$ with respect to Proposition V.4 is one where only the positive term is non-zero. Hence, it is enough to bound the expression above for positive $z$ :

$$
\left\|\sum_{y \in M} z(y) \otimes \operatorname{Con}\left(x_{i}\right)(y)\right\|_{\infty}=\sup _{|\psi\rangle \in \mathbb{C}^{Q^{2}}}\left\langle\psi\left|\sum_{y \in M} z(y) \otimes \operatorname{Con}\left(x_{i}\right)(y)\right| \psi\right\rangle=\sup _{C} \operatorname{Tr}\left[\sum_{y \in M} C z(y)^{T} C^{*} \operatorname{Con}\left(x_{i}\right)(y)\right]
$$

where the last supremum is over all operators $C$ on $Q$ with singular values equal to the Schmidt coefficients of $\psi$. Due to the properties of $x_{i}$

$$
\sum_{k \in N} \operatorname{Tr}_{Q}\left[C^{*} x_{i}(k) C\right] \leq 1 \quad \text { and } \quad C^{*} x_{i}(y) C \leq 2^{-k} C^{*} C
$$

which implies that $\rho_{i}(k)=C^{*} x_{i}(k) C$ defines a sub-normalized classical-quantum state on $N Q$ having min-entropy at least $k$. In order to have a valid state, we define

$$
\bar{\rho}_{i}=\rho_{i}+\left(1-\operatorname{Tr}\left[\rho_{i}\right]\right) \frac{\mathbb{1}}{Q} \otimes \frac{\mathbb{1}}{N} .
$$


As before, we can say that $\|\bar{\rho}\|_{(1 ; \infty)} \leq 2^{-k}+\frac{1}{N} \leq 2^{-k+1}$ and hence the min-entropy is at least $k-1$. It follows that $\operatorname{Con}\left(C^{*} x_{i} C\right) \leq \operatorname{Con} \bar{\rho}_{i}=\omega_{i}$ where $\omega_{i}$ is a state with $\varepsilon$-smooth min-entropy at least $k^{\prime}$. Hence, we find by Lemma VI.3

$$
\operatorname{Tr}\left[\sum_{y \in M} C z(y)^{T} C^{*} \operatorname{Con}\left(x_{i}\right)(y)\right]=\operatorname{Tr}\left[\sum_{y \in M} z\left(y^{T}\right) \operatorname{Con}\left(C^{*} x_{i} C\right)(y)\right] \leq \operatorname{Tr}\left[\sum_{y \in M} z(y)^{T} \omega_{i}(y)\right] \leq 2 \varepsilon
$$

\section{Graph Theory}

Proposition VI.5. Let Con $=\left\{f_{s}\right\}_{s \in D}$, [Con] as defined in $(30), G=(N, M, V, D)$ be the bipartite graph as defined in (39), and Dense $\left(G, 2^{k}, 2^{k^{\prime}}\right)$ be the optimal value of the quadratic program

$$
\begin{aligned}
& \operatorname{Dense}\left(G, 2^{k}, 2^{k^{\prime}}\right):=\text { maximize } \sum_{(x, y) \in V} f_{x} g_{y} \\
& \text { subject to } f_{x}, g_{y} \in\{0,1\} \\
& \sum_{x} f_{x} \leq 2^{k} \\
& \sum_{y} g_{y} \leq 2^{k^{\prime}}
\end{aligned}
$$

Then, we have

$$
\|[\text { Con }]: \cap\left\{2^{k} \ell_{\infty}, \ell_{1}\right\} \rightarrow \Sigma\left\{2^{k^{\prime}} \ell_{\infty}, \ell_{1}\right\} \| \leq \varepsilon \quad \Leftrightarrow \quad \operatorname{Dense}\left(G, 2^{k}, 2^{k^{\prime}}\right) \leq 2^{k+d} \varepsilon
$$

Proof. Using the fact that $\Sigma\left\{2^{k^{\prime}} \ell_{\infty}, \ell_{1}\right\}$ is the dual norm of the norm $\cap\left\{2^{-k^{\prime}} \ell_{1}, \ell_{\infty}\right\}$, we write

$$
\begin{aligned}
& \| \text { Con }: \cap\left\{2^{k} \ell_{\infty}, \ell_{1}\right\} \rightarrow \Sigma\left\{2^{k^{\prime}} \ell_{\infty}, \ell_{1}\right\} \| \\
& =\max \left\{\left|\sum_{y \in M} \operatorname{Con}(f)_{y} \cdot g_{y}\right|:\|f\|_{\cap\left\{2^{k} \ell_{\infty}, \ell_{1}\right\}} \leq 1,\|g\|_{\cap\left\{2^{-k^{\prime}} \ell_{1}, \ell_{\infty}\right\}} \leq 1\right\}
\end{aligned}
$$

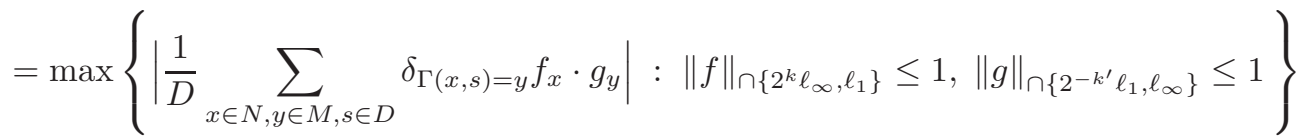

$$
\begin{aligned}
& =\frac{1}{D \cdot 2^{k}} \max \left\{\left|\sum_{x \in N, y \in M, s \in D} \delta_{\Gamma(x, s)=y} f_{x} \cdot g_{y}\right|:\|f\|_{\cap\left\{\ell_{\infty}, 2^{-k} \ell_{1}\right\}} \leq 1,\|g\|_{\cap\left\{2^{-k^{\prime}} \ell_{1}, \ell_{\infty}\right\}} \leq 1\right\} \text {, }
\end{aligned}
$$

where $f_{x}, g_{y}$ denote the components of the vectors $f \in \mathbb{R}^{N}, g \in \mathbb{R}^{M}$. Because the matrix elements of the tensor are all positive, we can restrict the maximization to vectors with positive entries. Then, the norm conditions on the vector $f$ translates into $0 \leq f_{x} \leq 1, \sum_{x} f_{x} \leq 2^{k}$. However, the extreme points of this convex sets are just the characteristic vectors of subsets of $N$ of size $2^{k}$. Due to the convex character of the objective function in both variables $f$ and $g$, we hence end up with the quadratic program as claimed.

\section{Bell inequalities}

As discussed in the review article [9] Bell inequalities and two-player games are the same, and in the following we will take the game perspective. In particular, we are interested in a two-player game such that its classical value is related to the bounded norm of the condenser as in Proposition II.5, and its entangled value relates to the completely bounded norm of the condenser as in Theorem II.6. The game is as follows. There are the two players Alice and Bob, and a referee. The bipartite graph $G=(N, M, V, D)$ as defined by the condenser Con $=\left\{f_{s}\right\}_{s \in D}$ via the neighbor 
function in (39) is known to all parties. First, the referee samples $\gamma=2^{n-k}$ elements $x_{1}, \ldots, x_{\gamma}$ out of $N$ uniformly at random (with replacement), and likewise $\gamma^{\prime}=2^{m-k^{\prime}}$ independent and uniformly chosen random entries $y_{1}, \ldots, y_{\gamma^{\prime}}$ out of $M$, as well as a value of the seed $s$ according to the uniform distribution. We collect these random indices into vectors $\vec{x} \in N^{\gamma}$ and $\vec{y} \in M^{\gamma^{\prime}}$; the questions for Alice and Bob, respectively. Alice and Bob then provide indices $1 \leq \alpha \leq \gamma$ and $1 \leq \beta \leq \gamma^{\prime}$ of these vectors as answers. They win if

$$
\Gamma\left(x_{\alpha}, s\right)=y_{\beta}
$$

We use the notation $\left(G ; 2^{k}, 2^{k^{\prime}}\right)$ for this game. A classical strategy amounts to a pair of deterministic mappings $f: N^{\gamma} \rightarrow\{1, \ldots, \gamma\}$ and $g: M^{\gamma^{\prime}} \rightarrow\left\{1, \ldots, \gamma^{\prime}\right\}$ (independent of the value of $s$ ), and the classical value of the game is 11

$$
\omega\left(G ; 2^{k}, 2^{k^{\prime}}\right):=\sup \left\{\mathbb{E}_{s} \mathbb{E}_{\vec{x}, \vec{y}} \Gamma[\vec{x}(f(\vec{x})), s ; \vec{y}(g(\vec{y}))]\right\},
$$

where the supremum is over all classical strategies, and

$$
\Gamma[x, s ; y]:= \begin{cases}1 & \text { for } \Gamma(x, s)=y \\ 0 & \text { otherwise }\end{cases}
$$

If Alice and Bob are allowed to share an entangled state of local dimension $Q$, they are not restricted to classical deterministic strategies. Instead, each player has a set of positive operator valued measures (POVM's), indexed by his questions, acting on his share of the entangled state. That is, Alice and Bob each have a set of positive operators in $\operatorname{Mat}_{Q}$ labeled by the questions $\vec{x}$ and $\vec{y}$ and possible outcomes $\alpha$ and $\beta$,

$$
\begin{array}{ll}
\hat{p}(\alpha ; \vec{x}) \geq 0 \quad \text { with } & \sum_{\alpha} \hat{p}(\alpha ; \vec{x}) \leq \mathbb{1} \\
\hat{q}(\beta ; \vec{y}) \geq 0 & \text { with } \quad \sum_{\beta} \hat{q}(\beta ; \vec{y}) \leq \mathbb{1}
\end{array}
$$

for all questions $\vec{x}$ and $\vec{y}$ (again independent of the value of $s$ ). Note that we allow for incomplete POVM's (they don't sum to the identity), since we can always include a dummy answer into the game, associated to the missing normalization, thereby not changing the value of the game. For fixed $Q \in \mathbb{N}$ we define

$$
\left.\omega_{Q}^{*}\left(G ; 2^{k}, 2^{k^{\prime}}\right):=\sup \left\{\mathbb{E}_{s} \mathbb{E}_{\vec{x}, \vec{y}} \sum_{\alpha, \beta} \Gamma[\vec{x}(\alpha)), s ; \vec{y}(\beta)\right] \cdot\langle\psi|\hat{p}(\alpha ; \vec{x}) \otimes \hat{q}(\beta ; \vec{y})| \psi\rangle\right\}
$$

where the supremum is over all bipartite pure state vectors $|\psi\rangle \in \mathbb{C}^{Q} \otimes \mathbb{C}^{Q}$, and all corresponding quantum strategies as in (181)-(182). The supremum over all bipartite pure state vectors just gives the operator norm and hence we have

$$
\left.\omega_{Q}^{*}\left(G ; 2^{k}, 2^{k^{\prime}}\right)=\sup \left\{\| \mathbb{E}_{s} \mathbb{E}_{\vec{x}, \vec{y}} \sum_{\alpha, \beta} \Gamma[\vec{x}(\alpha)), s ; \vec{y}(\beta)\right] \hat{p}(\alpha ; \vec{x}) \otimes \hat{q}(\beta ; \vec{y}) \|_{(\infty ; \infty)}\right\}
$$

Finally, the entangled value of the game is given by

$$
\omega^{*}\left(G ; 2^{k}, 2^{k^{\prime}}\right):=\sup _{Q \in \mathbb{N}} \omega_{Q}^{*}\left(G ; 2^{k}, 2^{k^{\prime}}\right)
$$

If we ask condensers only to be quantum-proof against $Q$-dimensional quantum side information the relevant quantity to bound becomes (as in Theorem II.6)

$$
\|[\text { Con }]\left\|_{Q, \cap \cdot \cap\left\{2^{k} \ell_{\infty}, \ell_{1}\right\} \rightarrow \Sigma \cdot \Sigma\left\{2^{k^{\prime}} \ell_{\infty}, \ell_{1}\right\}}:=\sup _{\substack{P_{N} \in \operatorname{Mat}_{Q}(N) \\\left\|P_{N}\right\|_{\cap \cdot \cap\left\{2^{k} \ell_{\infty}, \ell_{1}\right\} \leq 1}}}\right\|[\mathrm{Con}]\left(P_{N}\right) \|_{\Sigma \cdot \Sigma\left\{2^{k^{\prime}} \ell_{\infty}, \ell_{1}\right\}} .
$$

\footnotetext{
${ }^{11}$ Instead of deterministic functions $f$ and $g$, we could also allow for shared randomness, which does not increase the value of the game.
} 
For the proof of Theorem II.8 we will show that for any dimension $Q \in \mathbb{N}$,

$$
\omega_{Q}^{*}\left(G ; 2^{k}, 2^{k^{\prime}}\right) \leq 2^{-k^{\prime}} \cdot\|[\mathrm{Con}]\|_{Q, \cap \cdot \cap\left\{2^{k} \ell_{\infty}, \ell_{1}\right\} \rightarrow \Sigma \cdot \Sigma\left\{2^{k^{\prime}} \ell_{\infty}, \ell_{1}\right\}} \leq c \cdot \omega_{Q}^{*}\left(G ; 2^{k}, 2^{k^{\prime}}\right)
$$

and with this prove all the claims at once (by letting $Q=1$ for (45), $Q \rightarrow \infty$ for (46), or leaving $Q$ free). For the second inequality in (187) we start from elements $p \in \operatorname{Mat}_{Q}(N)$ and $q \in \operatorname{Mat}_{Q}(M)$, satisfying

$$
\sum_{x} p(x) \leq \frac{2^{k}}{4} \cdot \mathbb{1}, p(x) \leq \mathbb{1} \quad \text { and } \quad \sum_{y} q(y) \leq \frac{2^{k^{\prime}}}{4} \cdot \mathbb{1}, q(y) \leq \mathbb{1}
$$

and construct POVM's $\hat{p}(\alpha ; \vec{x})$ and $\hat{q}(\beta ; \vec{y})$ associated to the questions and answers of the game such that

$$
\frac{1}{64} \cdot \frac{4}{2^{k}} \cdot \frac{4}{2^{k^{\prime}}}\left\|\sum_{x, y} \mathbb{E}_{s} \Gamma[x, s ; y] p(x) \otimes q(y)\right\|_{(\infty ; \infty)} \leq\left\|\mathbb{E}_{s} \mathbb{E}_{\vec{x}, \vec{y}} \sum_{\alpha, \beta} \Gamma[\vec{x}(\alpha), s ; \vec{y}(\beta)] \hat{p}(\alpha ; \vec{x}) \otimes \hat{q}(\beta ; \vec{y})\right\|_{(\infty ; \infty)}
$$

Since the supremum of the left hand side over all such $q$ and $p$ is by operator space duality just $1 / 64$ times $\|$ Con $\|_{\text {cb }}$, and the statement follows by taking the expectation over the value of the seed $s$ on both sides. Conversely, the first inequality in (187) is proven by constructing operators satisfying the $\cap \cdot \cap$ norm estimates out of POVM elements.

The POVM's $\hat{q}(\beta ; \vec{y})$ and $\hat{p}(\alpha ; \vec{x})$ are built in a similar manner. Let $\vec{x}$ denote again the vector with entries corresponding to the choice of $\gamma$ independent random indices in $N$. For $0 \leq \alpha \leq \gamma, 1 \leq l \leq \alpha$ or $0 \leq \beta \leq \gamma^{\prime}, 1 \leq l^{\prime} \leq \beta$ we define for $p \in \operatorname{Mat}_{Q}(N)$ and $q \in \operatorname{Mat}_{Q}(M)$,

$$
r_{\vec{x}, l, \alpha}:=\prod_{l \leq k \leq \alpha}(\mathbb{1}-p[\vec{x}(k)])^{1 / 2}, \quad t_{\vec{y}, l^{\prime}, \beta}:=\prod_{l^{\prime} \leq k \leq \beta}(\mathbb{1}-q[\vec{y}(k)])^{1 / 2}
$$

as well as

$$
r_{\vec{x}, \alpha+1, \alpha}:=\mathbb{1}, \quad r_{\vec{x}, \alpha}:=r_{\vec{x}, 1, \alpha} \quad \text { and } \quad t_{\vec{y}, \beta+1, \beta}:=\mathbb{1}, \quad t_{\vec{y}, \beta}:=t_{\vec{y}, 1, \beta}
$$

The POVM's are then constructed as

$$
\hat{p}(\alpha ; \vec{x}):=r_{\vec{x}, \alpha-1}^{*} p[\vec{x}(\alpha)] r_{\vec{x}, \alpha-1} \quad \text { and } \quad \hat{q}(\beta ; \vec{y}):=t_{\vec{y}, \beta-1}^{*} q[\vec{y}(\beta)] t_{\vec{y}, \beta-1}
$$

The following lemma asserts that these are valid POVM's and also provides some estimates for remaining terms, to be used later on. It is a special case of [17, Lemma 6.7], but for the convenience of the reader we restate it, and provide an adapted proof in Appendix A.

Lemma VI.6. Let $p \in \operatorname{Mat}_{Q}(N)$, and $\alpha, \gamma, r_{\vec{x}, \alpha}$ as in (190)-(191). Then, we have

$$
\begin{aligned}
& \sum_{\alpha=1}^{\gamma} r_{\vec{x}, \alpha-1}^{*} p[\vec{x}(\alpha)] r_{\vec{x}, \alpha-1} \leq \mathbb{1} \\
& \sum_{\alpha=1}^{\gamma} \mathbb{E}_{x_{1}, \ldots, x_{\alpha-1}}\left(\mathbb{1}-r_{\vec{x}, \alpha-1}\right) \leq \frac{\gamma}{8} \cdot \mathbb{1} \\
& \sum_{\alpha=1}^{\gamma} \mathbb{E}_{x_{1}, \ldots, x_{\alpha-1}}\left[\mathbb{1}-r_{\vec{x}, \alpha-1}^{*}\right]\left[\mathbb{1}-r_{\vec{x}, \alpha-1}\right] \leq \frac{\gamma}{4} \cdot \mathbb{1} .
\end{aligned}
$$

Similar estimates hold for $q \in \operatorname{Mat}_{Q}(M)$ with the replacements $\alpha \mapsto \beta, \gamma \mapsto \gamma^{\prime}$, and $r_{\vec{x}, \alpha} \mapsto t_{\vec{y}, \beta}$ as defined in $(190)-$ (191).

Proof of Theorem II.8. We start with the direction game $\Longrightarrow$ condenser. The main idea of the proof is similar to the proof of [17, Proposition 6.9]. According to the rule

$$
t^{*} q t=q+\left(1-t^{*}\right) 1+q(1-t)-\left(1-t^{*}\right) q(1-t) \leq q+\left(1-t^{*}\right) 1+q(1-t)+\left(1-t^{*}\right) q(1-t)
$$


we split the following sum into 16 terms,

$$
\begin{aligned}
& \sum_{\alpha, \beta} \Gamma[\vec{x}(\alpha), s ; \vec{y}(\beta)] p[\vec{x}(\alpha)] \otimes q[\vec{y}(\beta)] \\
& \leq \sum_{\alpha, \beta} \Gamma[\vec{x}(\alpha), s ; \vec{y}(\beta)] r_{\vec{x}, \alpha-1}^{*} p[\vec{x}(\alpha)] r_{\vec{x}, \alpha-1} \otimes t_{\vec{y}, \beta-1}^{*} q[\vec{y}(\beta)] t_{\vec{y}, \beta-1} \\
& \quad+\sum_{\alpha, \beta} \Gamma[\vec{x}(\alpha), s ; \vec{y}(\beta)]\left[\mathbb{1}-r_{\vec{x}, \alpha-1}^{*}\right] p[\vec{x}(\alpha)] \otimes\left[\mathbb{1}-t_{\vec{y}, \beta-1}^{*}\right] q[\vec{y}(\beta)] \\
& \quad+\sum_{\alpha, \beta} \Gamma[\vec{x}(\alpha), s ; \vec{y}(\beta)] p[\vec{x}(\alpha)]\left[\mathbb{1}-r_{\vec{x}, \alpha-1}\right] \otimes q[\vec{y}(\beta)]\left[\mathbb{1}-t_{\vec{y}, \beta-1}\right] \\
& \quad+\ldots 12 \text { other terms } \\
& \quad+\sum_{\alpha, \beta} \Gamma[\vec{x}(\alpha), s ; \vec{y}(\beta)]\left[\mathbb{1}-r_{\vec{x}, \alpha-1}^{*}\right] p[\vec{x}(\alpha)]\left[\mathbb{1}-r_{\vec{x}, \alpha-1}\right] \otimes\left[\mathbb{1}-t_{\vec{y}, \beta-1}^{*}\right] q[\vec{y}(\beta)]\left[\mathbb{1}-t_{\vec{y}, \beta-1}\right] .
\end{aligned}
$$

We now take the expectation over the random choices $\vec{x}$ and $\vec{y}$ and the seed value $s$. The left hand side reduces to

$$
\begin{aligned}
\mathbb{E}_{s} \mathbb{E}_{\vec{x}, \vec{y}} \sum_{\alpha, \beta} \Gamma[\vec{x}(\alpha), s ; \vec{y}(\beta)] p[\vec{x}(\alpha)] \otimes q[\vec{y}(\beta)] & =\mathbb{E}_{s} \mathbb{E}_{x_{1}, \ldots, x_{\gamma}, y_{1}, \ldots, y_{\gamma^{\prime}}} \sum_{\alpha, \beta} \Gamma\left[x_{\alpha}, s ; y_{\beta}\right] p\left[x_{\alpha}\right] \otimes q\left[y_{\beta}\right] \\
& =\gamma \gamma^{\prime} \mathbb{E}_{s} \mathbb{E}_{x} \mathbb{E}_{y} \Gamma[x, s ; y] p(x) \otimes q(y) .
\end{aligned}
$$

The first term on the right hand side of (197) is the Bell (or game) operator which norm is equal to the value of the strategy given by the POVM's $\hat{q}(\beta ; \vec{y})$ and $\hat{p}(\alpha ; \vec{x})$. Let us examine the remaining terms. Expressions such as the second or third one are evaluated to

$$
\begin{aligned}
& \sum_{\alpha, \beta} \mathbb{E}_{s} \mathbb{E}_{x_{\alpha}} \mathbb{E}_{y_{\beta}}\left\{\Gamma[\vec{x}(\alpha), s ; \vec{y}(\beta)]\left(\mathbb{E}_{x_{1}, \ldots, x_{\alpha-1}}\left[\mathbb{1}-r_{\vec{x}, \alpha-1}^{*}\right]\right) p[\vec{x}(\alpha)] \otimes\left(\mathbb{E}_{y_{1}, \ldots, y_{\beta}-1}\left[\mathbb{1}-t_{\vec{y}, \beta-1}^{*}\right]\right) q[\vec{y}(\beta)]\right\} \\
& \leq \frac{\gamma \gamma^{\prime}}{8 \cdot 8}\left\|\mathbb{E}_{s} \mathbb{E}_{x} \mathbb{E}_{y} \Gamma[x, s ; y] p(x) \otimes q(y)\right\|_{(\infty ; \infty)} \cdot \mathbb{1},
\end{aligned}
$$

where we applied Lemma VI.6, (194), to the operators in the brackets. Note that we had to use that the estimate are independent of the seed value. Terms involving $\left[\mathbb{1}-r_{\ldots} ..\right]$ or its starred version on both sides of $p[\ldots]$ (and similarly those involving $[\mathbb{1}-t \ldots]$ on both sides of $q[\ldots])$ are estimated as follows

$$
\begin{aligned}
& \sum_{\alpha, \beta} \mathbb{E}_{s} \mathbb{E}_{x_{\alpha}} \mathbb{E}_{y_{\beta}}\left\{\Gamma[\vec{x}(\alpha), s ; \vec{y}(\beta)] \mathbb{E}_{x_{1}, \ldots, x_{\alpha-1}}\left\{\left[\mathbb{1}-r_{\vec{x}, \alpha-1}^{*}\right] p[\vec{x}(\alpha)]\left[\mathbb{1}-r_{\vec{x}, \alpha-1}\right]\right\} \otimes \mathbb{E}_{y_{1}, \ldots, y_{\beta-1}}\left\{\left[\mathbb{1}-t_{\vec{y}, \beta-1}^{*}\right] q[\vec{y}(\beta)]\left[\mathbb{1}-t_{\vec{y}, \beta-1}\right]\right\}\right\} \\
& \leq\left\|\mathbb{E}_{s} \mathbb{E}_{x} \mathbb{E}_{y} \Gamma[x, s ; y] p(x) \otimes q(s, y)\right\|_{(\infty ; \infty)} \cdot\left\|\sum_{\alpha} \mathbb{E}_{x_{1}, \ldots, x_{\alpha-1}}\left[\mathbb{1}-r_{\vec{x}, \alpha-1}^{*}\right]\left[\mathbb{1}-r_{\vec{x}, \alpha-1}\right]\right\|_{(\infty ; \infty)} \\
& \quad \cdot \max _{s}\left\|\sum_{\beta} \mathbb{E}_{y_{1}, \ldots, y_{\beta-1}}\left[\mathbb{1}-t_{s, \vec{y}, \beta-1}^{*}\right]\left[\mathbb{1}-t_{\vec{y}, \beta-1}\right]\right\|_{(\infty ; \infty)} \cdot \mathbb{1} \\
& \leq \frac{\gamma \gamma^{\prime}}{4 \cdot 4}\left\|\mathbb{E}_{s} \mathbb{E}_{x} \mathbb{E}_{y} \Gamma[x, s ; y] p(x) \otimes q(y)\right\|_{(\infty ; \infty)} \cdot \mathbb{1}
\end{aligned}
$$

Here we used that for completely positive maps $\phi$ and positive operators $a$ it holds that $\phi(a) \leq\|a\| \phi(\mathbb{1})$, which we applied to the cp maps given by the Kraus operators $\mathbb{1}-r_{\vec{x}, \alpha-1}$ and $\mathbb{1}-t_{\vec{y}, \beta-1}$. The estimate then followed by applying Lemma VI.6, (195), and again using that the estimate are independent of the seed value. Estimating all cross terms according to the two strategies leads to the final estimate

$$
\begin{aligned}
\frac{1}{4} \cdot 2^{-k-k^{\prime}}\left\|\sum_{x, y} \mathbb{E}_{s} \Gamma[x, s ; y] p(x) \otimes q(y)\right\|_{(\infty ; \infty)} & =\gamma \gamma^{\prime}\left\|\mathbb{E}_{s} \mathbb{E}_{x} \mathbb{E}_{y} \Gamma[x, s ; y] p(x) \otimes q(y)\right\|_{(\infty ; \infty)} \\
& \leq\left\|\sum_{\alpha, \beta} \mathbb{E}_{s} \mathbb{E}_{\vec{x}, \vec{y}} \Gamma[\vec{x}(\alpha), s ; \vec{y}(\beta)] \hat{p}(\alpha ; \vec{x}) \otimes \hat{q}(\beta ; \vec{y})\right\|_{(\infty ; \infty)} .
\end{aligned}
$$

For the converse part, let $\hat{q}(\beta ; \vec{y})$ and $\hat{p}(\alpha ; \vec{x})$ be arbitrary quantum strategies. We perform the following transformation

$\mathbb{E}_{s} \mathbb{E}_{\vec{x}, \vec{y}} \sum_{\alpha, \beta} \Gamma[\vec{x}(\alpha), s ; \vec{j}(\beta)] \hat{p}(\alpha ; \vec{x}) \otimes \hat{q}(\beta ; \vec{y})=\sum_{x^{\prime} \in N, y^{\prime} \in M} \mathbb{E}_{s} \Gamma[x, s ; y] \sum_{\alpha} \mathbb{E}_{\vec{x}}\left[\delta_{\vec{x}(\alpha)=x^{\prime}} \hat{p}(\alpha ; \vec{x})\right] \otimes \sum_{\beta} \mathbb{E}_{\vec{y}}\left[\delta_{\vec{y}(\beta)=y^{\prime}}(\beta ; \vec{y})\right]$ 
We now show that the collection of operators

$$
p\left(x^{\prime}\right)=\sum_{\alpha} \mathbb{E}_{\vec{x}}\left[\delta_{\vec{x}(\alpha)=x^{\prime}} \hat{p}(\alpha ; \vec{x})\right] \quad \text { and } \quad q\left(y^{\prime}\right)=\sum_{\beta} \mathbb{E}_{\vec{y}}\left[\delta_{\vec{y}(\beta)=y^{\prime}} \hat{q}(\beta ; \vec{y})\right]
$$

satisfy the $\cap \cdot \cap$-norm estimates

$$
\sum_{x^{\prime}} p\left(x^{\prime}\right) \leq \mathbb{1} \quad \text { and } \quad p\left(x^{\prime}\right) \leq 2^{-k} \mathbb{1} \quad \text { plus } \quad \sum_{y^{\prime}} q\left(y^{\prime}\right) \leq \mathbb{1} \quad \text { and } \quad q\left(y^{\prime}\right) \leq 2^{-k^{\prime}} \mathbb{1} .
$$

Hence, we have

$$
\begin{aligned}
& \left\|\mathbb{E}_{s} \mathbb{E}_{\vec{x}, \vec{y}} \sum_{\alpha, \beta} \Gamma[\vec{x}(\alpha), s ; \vec{j}(\beta)] \hat{p}(\alpha ; \vec{x}) \otimes \hat{q}(\beta ; \vec{y})\right\|_{(\infty ; \infty)} \\
& \leq \sup \left\{\left\|\sum_{x, y} \mathbb{E}_{s} \Gamma[x, s ; y] p(x) \otimes q(y)\right\|_{(\infty ; \infty)}:\|p\|_{\cap \cdot \cap\left\{2^{k} \ell_{\infty}, \ell_{1}\right\}} \leq 1,\|q\|_{\cap \cdot \cap\left\{2^{k^{\prime}} \ell_{\infty}, \ell_{1}\right\}} \leq 1\right\},
\end{aligned}
$$

and again operator space duality provides the last argument.

To show the first set of estimates in (207), note

$$
\sum_{x^{\prime}} \sum_{\alpha} \mathbb{E}_{\vec{x}}\left[\delta_{\vec{x}(\alpha)=x^{\prime}} \hat{p}(\alpha ; \vec{x})\right]=\sum_{\alpha} \mathbb{E}_{\vec{x}}\left[\sum_{x^{\prime}} \delta_{\vec{x}(\alpha)=x^{\prime}} \hat{p}(\alpha ; \vec{x})\right]=\sum_{\alpha} \hat{p}(\alpha ; \vec{x}) \leq \mathbb{1},
$$

and similarly $\sum_{y^{\prime}, \beta} \mathbb{E}_{\vec{y}}\left[\delta_{\vec{y}(\beta)=y^{\prime}} \hat{q}(\beta ; \vec{y})\right] \leq \mathbb{1}$. Moreover, we have $\hat{p}(\alpha ; \vec{x}) \leq \mathbb{1}$ and hence

$$
\sum_{\alpha} \mathbb{E}_{\vec{x}}\left[\delta_{\vec{x}(\alpha)=x^{\prime}} \hat{p}(\alpha ; \vec{x})\right] \leq \sum_{\alpha} \mathbb{P}\left[x_{\alpha}=x^{\prime}\right] \leq \frac{\gamma}{N} \mathbb{1}=2^{-k} \mathbb{1},
$$

and again similarly $\mathbb{E}_{\vec{y}}\left[\delta_{\vec{y}(\beta)=y^{\prime}} \hat{q}(\beta ; \vec{y})\right] \leq 2^{-k^{\prime}}$

\section{ACKNOWLEDGMENTS}

We acknowledge discussions with Matthias Christandl, Fabian Furrer, Patrick Hayden, Christopher Portmann, Renato Renner, Oleg Szehr, Marco Tomamichel, Thomas Vidick, Stephanie Wehner, Reinhard Werner, and Andreas Winter. Part of this work was done while OF and VBS were visiting the Institute for Quantum Information and Matter at Caltech and we would like to thank John Preskill and Thomas Vidick for their hospitality. Most of this work was done while OF was at ETH Zurich supported by the the European Research Council grant No. 258932. Additional funding was provided by the EU under the project "Randomness and Quantum Entanglement" (RAQUEL). VBS was supported by an ETH postdoctoral fellowship.

\section{Appendix A: Missing proofs}

Theorem A.1 (Grothendieck's inequality). For any real matrix $\left\{A_{i j}\right\}$, we have

$$
\max \left\{\sum_{i, j} A_{i j} \vec{a}_{i} \cdot \vec{b}_{j}:\left\|\vec{a}_{i}\right\|_{2} \leq 1,\left\|\vec{b}_{j}\right\|_{2} \leq 1\right\} \leq K_{G} \cdot \max \left\{\sum_{i, j} A_{i j} a_{i} b_{j}: a_{i}, b_{j} \in \mathbb{R},\left|a_{i}\right| \leq 1,\left|b_{j}\right| \leq 1\right\} .
$$

Proposition A.2. On $\operatorname{Mat}_{Q N}$ the norm $\|\cdot\|_{(1 ; \infty)}$ is dual to the norm $\|\cdot\|_{(\infty ; 1)}$.

Proof. For $A \in \operatorname{Mat}_{Q N}$ we calculate

$$
\begin{aligned}
\|A\|_{(1 ; \infty)^{*}} & =\sup \left\{\operatorname{Tr}\left[B^{*} A\right]:\|B\|_{(1 ; \infty)} \leq 1\right\} \\
& =\sup \left\{\operatorname{Tr}\left[B^{*} A\right]: B=\left(D_{1} \otimes \mathbb{1}_{N}\right) C\left(D_{2} \otimes \mathbb{1}_{N}\right),\left\|D_{1}\right\|_{2},\left\|D_{2}\right\|_{2} \leq 1,\|C\|_{(\infty ; \infty)} \leq 1\right\} \\
& =\sup \left\{\operatorname{Tr}\left[C^{*}\left(D_{1}^{*} \otimes \mathbb{1}_{N}\right) A\left(D_{2}^{*} \otimes \mathbb{1}_{N}\right)\right],\left\|D_{1}\right\|_{2},\left\|D_{2}\right\|_{2} \leq 1,\|C\|_{(\infty ; \infty)} \leq 1\right\} \\
& =\sup \left\{\left\|\left(D_{2}^{*} \otimes \mathbb{1}_{N}\right) A\left(D_{1}^{*} \otimes \mathbb{1}_{N}\right)\right\|_{(1 ; 1)},\left\|D_{1}\right\|_{2},\left\|D_{2}\right\|_{2} \leq 1\right\} \\
& =\|A\|_{(\infty ; 1)},
\end{aligned}
$$

where we have used that $\|\cdot\|_{(\infty ; \infty)}$ is dual to $\|\cdot\|_{(1 ; 1)}$. 
Proposition A.3. For $\rho_{Q N} \in \mathcal{S}(Q N)$ we have $\left\|\rho_{Q N}\right\|_{(1 ; \infty)}=2^{-H_{\min }(N \mid Q)_{\rho}}$.

Proof. This is basically proven in [13] but we reproduce the argument here for convenience. Let $\sigma_{Q} \in \mathcal{S}(Q) \subset$ Mat $_{Q}$ be such that $2^{-H_{\min }(N \mid Q)_{\rho}}=\left\|\left(\sigma_{Q}^{-1 / 2} \otimes \mathbb{1}_{N}\right) \rho_{Q N}\left(\sigma_{Q}^{-1 / 2} \otimes \mathbb{1}_{N}\right)\right\|_{(\infty ; \infty)}$. Then, we have

$$
\left\|\rho_{Q N}\right\|_{(1 ; \infty)} \leq\left\|\sigma_{Q}^{1 / 2}\right\|_{2}\left\|\left(\sigma_{Q}^{-1 / 2} \otimes \mathbb{1}_{N}\right) \rho_{Q N}\left(\sigma_{Q}^{-1 / 2} \otimes \mathbb{1}_{N}\right)\right\|_{(\infty ; \infty)}\left\|\sigma_{Q}^{1 / 2}\right\|_{2}=2^{-H_{\min }(N \mid Q)_{\rho}}
$$

For the other direction we will show that for $\rho_{Q N} \geq 0$,

$$
\left\|\rho_{Q N}\right\|_{(1 ; \infty)}=\inf \left\{\|A\|_{2}\left\|\omega_{Q N}\right\|_{(\infty ; \infty)}\|A\|_{2}: \rho_{Q N}=\left(A \otimes \mathbb{1}_{N}\right) \omega_{Q N}\left(A \otimes \mathbb{1}_{N}\right) ; A \in \operatorname{Mat}_{Q}, A \geq 0\right\},
$$

from which the claim follows.

We prove the similar statement for the dual norm $\|\cdot\|_{(\infty ; 1)}$ first. For that let $X \geq 0$ and using Hölder's inequality we get

$$
\left\|A X B^{*}\right\|_{1}=\left\|A \sqrt{X} \sqrt{X} B^{*}\right\|_{1} \leq\|A \sqrt{X}\|_{2}\left\|\sqrt{X} B^{*}\right\|_{2} \leq \max \left\{\left\|A X A^{*}\right\|_{1},\left\|B X B^{*}\right\|_{1}\right\} .
$$

By performing a polar decomposition of $A$ and using the unitary invariance of the norm $\|\cdot\|_{1}$, we get that

$$
\|X\|_{(\infty ; 1)}=\sup \left\{\|A X A\|_{1}: A \in \operatorname{Mat}_{Q}, A \geq 0,\|A\|_{2} \leq 1\right\} .
$$

By the same arguments which show that the norm $\|\cdot\|_{(\infty ; 1)}$ is dual to the norm $\|\cdot\|_{(1 ; \infty)}$ (Proposition A.2), this then implies (A8).

Lemma A.4. Let $u: \operatorname{Mat}_{N} \rightarrow \operatorname{Mat}_{Q}, z \in \operatorname{Mat}_{Q N}$, as well as $a=\sum_{i j} a_{i, j}|i\rangle \otimes|j\rangle, b=\sum_{i, j} b_{i j}|i\rangle \otimes|j\rangle$ vectors in $\mathbb{C}^{Q^{2}}$. Then, we have

$$
\left\langle b\left|\left(\mathbb{1}_{Q} \otimes u\right)(z)\right| a\right\rangle=\operatorname{Tr}\left[J(u)\left(A^{T} \otimes \mathbb{1}_{N}\right) z^{T}\left(\bar{B} \otimes \mathbb{1}_{N}\right)\right],
$$

where $J(u)=\left(\sum_{k, k^{\prime}} u\left(|k\rangle\left\langle k^{\prime}\right|\right) \otimes|k\rangle\left\langle k^{\prime}\right|\right), A=\sum_{i, j} a_{i j}|i\rangle\langle j|$, and $B=\sum_{i, j} b_{i j}|i\rangle\langle j|$.

Proof. We write

$$
\begin{aligned}
\left\langle b\left|\left(\mathbb{1}_{Q} \otimes u\right)(z)\right| a\right\rangle & =\sum_{i, i^{\prime}, j, j^{\prime}} \operatorname{Tr}\left[\left(\mathbb{1}_{Q} \otimes u\right)(z) a_{i j} \bar{b}_{i^{\prime} j^{\prime}}|i\rangle\left\langle i^{\prime}|\otimes| j\right\rangle\left\langle j^{\prime}\right|\right] \\
& =\sum_{i, i^{\prime}, j, j^{\prime}, k, k^{\prime}} z_{i^{\prime} i k k^{\prime}} a_{i j} \bar{b}_{i^{\prime} j^{\prime}} \operatorname{Tr}\left[|i\rangle\left\langle i^{\prime}\left|\otimes u\left(|k\rangle\left\langle k^{\prime}\right|\right)\right| i^{\prime}\right\rangle\langle i|\otimes| j\rangle\left\langle j^{\prime}\right|\right] \\
& =\sum_{i, i^{\prime}, j, j^{\prime}, k, k^{\prime}} z_{i^{\prime} i k k^{\prime}} a_{i j} \bar{b}_{i^{\prime} j^{\prime}} \operatorname{Tr}\left[x|j\rangle\left\langle j^{\prime}|\otimes| k^{\prime}\right\rangle\langle k|\right] \\
& =\sum_{i, i^{\prime}, k, k^{\prime}} z_{i^{\prime} i k k^{\prime}} \operatorname{Tr}\left[J(u)\left(\sum_{j} a_{i j}|j\rangle\right)\left(\sum_{j^{\prime}} \bar{b}_{i^{\prime} j^{\prime}}\left\langle j^{\prime}\right|\right) \otimes\left|k^{\prime}\right\rangle\langle k|\right] \\
& =\sum_{i, i^{\prime}, k, k^{\prime}} z_{i^{\prime} i k k^{\prime}} \operatorname{Tr}\left[J(u) A^{T}|i\rangle\left\langle i^{\prime}|\bar{B} \otimes| k^{\prime}\right\rangle\langle k|\right] \\
& =\operatorname{Tr}\left[\left(\bar{B} \otimes \mathbb{1}_{N}\right) J(u)\left(A^{T} \otimes \mathbb{1}_{N}\right) z^{T}\right] .
\end{aligned}
$$

Lemma VI.6. Let $p \in \operatorname{Mat}_{Q}(N)$, and $\alpha, \gamma, r_{\vec{x}, \alpha}$ as in (190)-(191). Then, we have

$$
\begin{aligned}
& \sum_{\alpha=1}^{\gamma} r_{\vec{x}, \alpha-1}^{*} p[\vec{x}(\alpha)] r_{\vec{x}, \alpha-1} \leq \mathbb{1} \\
& \sum_{\alpha=1}^{\gamma} \mathbb{E}_{x_{1}, \ldots, x_{\alpha-1}}\left(\mathbb{1}-r_{\vec{x}, \alpha-1}\right) \leq \frac{\gamma}{8} \cdot \mathbb{1} \\
& \sum_{\alpha=1}^{\gamma} \mathbb{E}_{x_{1}, \ldots, x_{\alpha-1}}\left[\mathbb{1}-r_{\vec{x}, \alpha-1}^{*}\right]\left[\mathbb{1}-r_{\vec{x}, \alpha-1}\right] \leq \frac{\gamma}{4} \cdot \mathbb{1} .
\end{aligned}
$$

Similar estimates hold for $q \in \operatorname{Mat}_{Q}(M)$ with the replacements $\alpha \mapsto \beta, \gamma \mapsto \gamma^{\prime}$, and $r_{\vec{x}, \alpha} \mapsto t_{\vec{y}, \beta}$ as defined in (190)(191). 
Proof. For the first property, note that

$$
\begin{aligned}
\mathbb{1}-\sum_{\alpha} r_{\vec{x}, \alpha-1}^{*} p[\vec{x}(\alpha)] r_{\vec{x}, \alpha-1} & =\mathbb{1}-p\left[x_{1}\right]-\sum_{\alpha=2} r_{\vec{x}, \alpha-1}^{*} p[\vec{x}(\alpha)] r_{\vec{x}, \alpha-1} \\
& =\left(\mathbb{1}-p\left[x_{1}\right]\right)^{1 / 2}\left(\mathbb{1}-\sum_{\alpha=2} r_{\vec{x}, 2, \alpha-1}^{*} p[\vec{x}(\alpha)] r_{\vec{x}, 2, \alpha-1}\right)\left(\mathbb{1}-p\left[x_{1}\right]\right)^{1 / 2} \\
& =\cdots=r_{\vec{x}, \gamma}^{*} r_{\vec{x}, \gamma} \\
& \geq 0 .
\end{aligned}
$$

For the second property, it follows from $1-\sqrt{1-t} \leq t, t \in[0,1]$ that $\mathbb{1}-(\mathbb{1}-p(k))^{1 / 2} \leq p(k)$ and hence also $\left(1-\frac{1}{4 \gamma}\right) \mathbb{1} \leq \frac{1}{N} \sum_{x}(\mathbb{1}-p(x))^{1 / 2}$. We then have

$$
\begin{aligned}
\sum_{\alpha}^{\gamma} \mathbb{E}_{x_{1}, \ldots, x_{\alpha-1}}\left(\mathbb{1}-r_{\vec{x}, \alpha-1}\right)=\sum_{\alpha=1}^{\gamma}\left(\mathbb{1}-\mathbb{E}_{x_{1}} \mathbb{E}_{x_{2}} \ldots \mathbb{E}_{x_{\alpha-1}} r_{\vec{x}, \alpha-1}\right) & =\sum_{\alpha=1}^{\gamma}\left(\mathbb{1}-\left(\frac{1}{N} \sum_{x}(\mathbb{1}-p(x))^{1 / 2}\right)^{\alpha-1}\right) \\
& \leq \sum_{\alpha=1}^{\gamma}\left(1-\left(1-\frac{1}{4 \gamma}\right)^{\alpha-1}\right) \mathbb{1} \\
& \leq \gamma\left(4\left(1-\frac{1}{4 \gamma}\right)^{\gamma}-3\right) \mathbb{1} \\
& \leq \gamma\left(4 e^{-1 / 4}-3\right) \mathbb{1} \\
& \leq \frac{\gamma}{8} \mathbb{1} .
\end{aligned}
$$

The last assertion follows by

$$
\begin{aligned}
& \sum_{\alpha} \mathbb{E}_{x_{1}, \ldots, x_{\alpha-1}}\left[\mathbb{1}-r_{\vec{x}, \alpha-1}^{*}\right]\left[\mathbb{1}-r_{\vec{x}, \alpha-1}\right] \\
& =\mathbb{1}-\sum_{\alpha} \mathbb{E}_{x_{1}, \ldots, x_{\alpha-1}}\left[r_{\vec{x}, \alpha-1}^{*}\right]-\sum_{\alpha} \mathbb{E}_{x_{1}, \ldots, x_{\alpha-1}}\left[r_{\vec{x}, \alpha-1}\right]+\sum_{\alpha} \mathbb{E}_{x_{1}, \ldots, x_{\alpha-1}}\left[r_{\vec{x}, \alpha-1}^{*} r_{\vec{x}, \alpha-1}\right] \\
& \leq 2\left(\mathbb{1}-\left(\frac{1}{N} \sum_{x}(\mathbb{1}-p(x))^{1 / 2}\right)^{\alpha-1}\right),
\end{aligned}
$$

since $r_{\vec{x}, \alpha-1}^{*} r_{\vec{x}, \alpha-1} \leq \mathbb{1}$. The proof for terms involving $t$ 's and $q$ 's is identical.

\section{Appendix B: Haagerup tensor product and intersection norms}

We denote the Haagerup tensor product by $\otimes_{h}$ and refer to [28, Chapter 5] for details on this tensor product. The calculations we perform here are based on a few simpler properties. For operator spaces $E$ and $F$ of dimension $N$, we have

$$
\|x\|_{E \otimes_{h} F}=\inf \left\{\| \sum_{i}|0\rangle\left\langle i\left|\otimes a_{i}\left\|_{M_{N}(E)} \cdot\right\| \sum_{i}\right| i\right\rangle\langle 0| \otimes b_{i} \|_{M_{N}(F)}: x=\sum_{i=1}^{N} a_{i} \otimes b_{i}\right\} .
$$

Using the min tensor product notation (see [28, Chapter 2] for a definition), the norm $M_{N}(E)$ is written as $M_{N} \otimes_{\min } E$. As only the first row of the matrix is used, we can write the norm as $R_{N} \otimes_{\min } E$.

$$
\|x\|_{E \otimes_{h} F}=\inf \left\{\left\|\sum_{i}\left\langle i\left|\otimes a_{i}\left\|_{R_{N} \otimes_{\min } E} \cdot\right\| \sum_{i}\right| i\right\rangle \otimes b_{i}\right\|_{C_{N} \otimes_{\min } F}: x=\sum_{i=1}^{N} a_{i} \otimes b_{i}\right\} .
$$

Recall that we previously mentioned the column and row operator spaces $C_{N}$ and $R_{N}$. They are simply defined by embedding a vector $\mathbb{C}^{N}$ as a column or row of a matrix in $\operatorname{Mat}_{N}$. So we have for $x=\sum_{i j k} x_{i j k}|i\rangle\langle j|\otimes| k\rangle \in \operatorname{Mat}_{Q}(N)$,

$$
\|x\|_{M_{Q}\left(C_{N}\right)}=\| \sum_{i j k} x_{i j k}|i\rangle\langle j|\otimes| k\rangle\langle 0| \|_{(\infty ; \infty)} \quad \text { and } \quad\|x\|_{M_{Q}\left(R_{N}\right)}=\| \sum_{i j k} x_{i j k}|i\rangle\langle j|\otimes| 0\rangle\langle k| \|_{(\infty ; \infty)} .
$$


In order to prove Proposition B.1, we use the following equalities equalities for norms (they are actually operator space equalities called complete isometries)

$$
\begin{aligned}
& C_{N} \otimes_{h} E=C_{N} \otimes_{\min } E \\
& E \otimes_{h} R=E \otimes_{\min } R \\
& C_{N} \otimes_{\min } R_{N}=M_{N} \\
& M_{Q}(E)=C_{Q} \otimes_{h} E \otimes_{h} R_{Q} \\
& M_{Q} \otimes_{\min } \cap\{E, F\}=\cap\left\{M_{Q} \otimes_{\min } E, M_{Q} \otimes_{\min } F\right\} .
\end{aligned}
$$

Proposition B.1. The operator space $\cap\left\{2^{k} \ell_{\infty}, \ell_{1}\right\}$ is completely isomorphic to the operator space $\cap\left\{\sqrt{2^{k}} C_{N}, R_{N}\right\} \otimes_{h}$ $\cap\left\{\sqrt{2^{k}} R_{N}, C_{N}\right\}=: G$.

Proof. Let $x \in \operatorname{Mat}_{Q} \otimes \operatorname{Mat}_{N}$ with $x=\sum_{i j k l} x_{i j k l}|i\rangle\langle j|\otimes| k\rangle\langle l|$. Our objective is to compute $\|x\|_{M_{Q}(G)}$. Using the rules for Haagerup tensor product, we can write this norm as follows:

$$
\begin{aligned}
M_{Q}(G) & =C_{Q} \otimes_{h} \cap\left\{\sqrt{2^{k}} C_{N}, R_{N}\right\} \otimes_{h} \cap\left\{\sqrt{2^{k}} R_{N}, C_{N}\right\} \otimes_{h} R_{Q} \\
& =C_{Q} \otimes_{\min } \cap\left\{\sqrt{2^{k}} C_{N}, R_{N}\right\} \otimes_{h} \cap\left\{\sqrt{2^{k}} R_{N}, C_{N}\right\} \otimes_{\min } R_{Q} \\
& =\cap\left\{\sqrt{2^{k}} C_{Q} \otimes_{\min } C_{N}, C_{Q} \otimes_{\min } R_{N}\right\} \otimes_{h} \cap\left\{\sqrt{2^{k}} R_{N} \otimes_{\min } R_{Q}, C_{N} \otimes_{\min } R_{Q}\right\} \\
& =\cap\left\{\sqrt{2^{k}} C_{Q N}, C_{Q} \otimes_{\min } R_{N}\right\} \otimes_{h} \cap\left\{\sqrt{2^{k}} R_{N Q}, C_{N} \otimes_{\min } R_{Q}\right\} .
\end{aligned}
$$

Then considering the definition (B1), it will involve the norms

$$
R_{Q N} \otimes_{\min } \cap\left\{\sqrt{2^{k}} C_{Q N}, C_{Q} \otimes_{\min } R_{N}\right\}=\cap\left\{\sqrt{2^{k}} M_{Q N}, R_{Q N} \otimes_{\min } C_{Q} \otimes_{\min } R_{N}\right\},
$$

and

$$
C_{Q N} \otimes_{\min } \cap\left\{\sqrt{2^{k}} R_{N Q}, C_{N} \otimes_{\min } R_{Q}\right\}=\cap\left\{\sqrt{2^{k}} M_{Q N}, C_{Q N} \otimes_{\min } C_{N} \otimes_{h} R_{Q}\right\} .
$$

Seeing $x$ as an element of $\mathbb{C}^{Q N} \otimes \mathbb{C}^{Q N}$, we have using the definition (B2)

$$
\begin{aligned}
&\|x\|_{M_{Q}(G)}=\inf \{ \max \left\{\sqrt{2^{k}} \| \sum_{i k p} a_{i k}(p)|i\rangle|k\rangle\left\langle p\left|\left\|_{(\infty ; \infty)},\right\| \sum_{i k p} a_{i k}(p)\right| i\right\rangle\langle k|\langle p| \|_{\infty}\right\} \\
&\left.\cdot \max \left\{\sqrt{2^{k}} \| \sum_{j l p} b_{j l}^{*}(p)|p\rangle\left\langle j\left|\left\langle l\left|\left\|_{(\infty ; \infty)},\right\| \sum_{j l p} b_{j l}^{*}(p)\right| p\right\rangle\right| j\right\rangle\langle l| \|_{\infty}\right\}: x=\sum_{p=1}^{N Q} a(p) \otimes b(p)\right\} .
\end{aligned}
$$

We have used the notation $a(p)=\sum_{i k} a_{i k}(p)|i\rangle|k\rangle$ and $b(p)=\sum_{j l} b_{j l}^{*}(p)\langle j|\langle l|$. If we define $a=\sum_{i k p} a_{i k}(p)|i\rangle|k\rangle\langle p|$ and $b=\sum_{j l p} b_{j l}(p)|j\rangle|l\rangle\langle p|$, then $x=\sum_{p} a(p) \otimes b(p)$ becomes $x=a b^{*}$. We then find exactly the expression in (81).

We end this appendix with an identification of the dual space of $\cap \cdot \cap$. Since the Haagerup tensor product is self dual [28, Chapter 5], Proposition B.1 is conveniently applied. First, note that by the discussion on intersection norms for operator spaces in the previous section, the dual space of $\cap\left\{\sqrt{2^{k}} C_{N}, R_{N}\right\}$ is equal to $\Sigma\left\{2^{-k / 2} R_{N}, C_{N}\right\}$, since the operator space dual of the row operator space the te column space and vice versa.

Corollary B.2. The operator space dual of $\cap \cdot \cap$ is the operator space $\Sigma\left\{2^{-k / 2} R_{N}, C_{N}\right\} \otimes_{h} \Sigma\left\{2^{-k / 2} R_{N}, C_{N}\right\}=$ $2^{-k}\left(\Sigma\left\{R_{N}, \sqrt{2^{k}} C_{N}\right\} \otimes_{h} \Sigma\left\{R_{N}, \sqrt{2^{k}} C_{N}\right\}\right)$.

[1] A. Ben-Aroya and A. Ta-Shma. Better short-seed quantum-proof extractors. Theoretical Computer Science, 419:17-25, 2012. DOI: $10.1016 / j \cdot t c s .2011 .11 .036$.

[2] C. H. Bennett, G. Brassard, C. Crépeau, and U. M. Maurer. Generalized privacy amplification. Information Theory, IEEE Transactions on, 41(6):1915-1923, 1995.

[3] C. H. Bennett, G. Brassard, and J.-M. Robert. Privacy amplification by public discussion. SIAM journal on Computing, 17(2):210-229, 1988. 
[4] M. Berta, O. Fawzi, V. B. Scholz, and O. Szehr. Variations on classical and quantum extractors. In Information Theory (ISIT), 2014 IEEE International Symposium on, pages 1474-1478, 2014. DOI: 10.1109/ISIT.2014.6875078.

[5] M. Berta, O. Fawzi, and S. Wehner. Quantum to classical randomness extractors. In Advances in Cryptology - CRYPTO 2012, volume 7417 of LNCS, pages 776-793. Springer, 2012. DOI : 10.1007/978-3-642-32009-5_45.

[6] M. Berta, O. Fawzi, and S. Wehner. Quantum to classical randomness extractors. Information Theory, IEEE Transactions on, 60:1168-1192, 2014. DOI: 10.1109/TIT.2013.2291780.

[7] A. Bhaskara, V. Guruswami, M. Charikar, A. Vijayaraghavan, and Y. Zhou. Polynomial integrality gaps for strong SDP relaxations of Densest k-subgraph. In SODA '12: Proceedings of the Twenty-Third Annual ACM-SIAM Symposium on Discrete Algorithms, 2012. Available online: http://dl.acm.org/citation.cfm?id=2095116.2095150.

[8] F. G. Brandao, R. Ramanathan, A. Grudka, K. Horodecki, M. Horodecki, and P. Horodecki. Robust device-independent randomness amplification with few devices. 2013. Available online: http://arxiv.org/abs/1310.4544.

[9] N. Brunner, D. Cavalcanti, S. Pironio, V. Scarani, and S. Wehner. Bell nonlocality. Reviews of Modern Physics, 86:419-478, 2014. DOI : 10.1103/RevModPhys.86.419.

[10] K.-M. Chung, Y. Shi, and X. Wu. Physical randomness extractors. 2014. Available online: http://arxiv.org/abs/1402.4797.

[11] M. Coudron and H. Yuen. Infinite randomness expansion and amplification with a constant number of devices. In Proceedings of the 46th Annual ACM Symposium on Theory of Computing, STOC '14, pages 427-436. ACM, 2014. Available online: http://dl.acm.org/citation. cfm?doid=2591796.2591873.

[12] A. De, C. Portmann, T. Vidick, and R. Renner. Trevisan's extractor in the presence of quantum side information. SIAM Journal on Computing, 41:915-940, 2012. DOI: 10.1137/100813683.

[13] I. Devetak, M. Junge, C. King, and M. B. Ruskai. Multiplicativity of completely bounded p-norms implies a new additivity result. Communications in mathematical physics, 266:37-63, 2006. DOI: 10.1007/s00220-006-0034-0.

[14] F. Dupuis, O. Fawzi, and S. Wehner. Achieving the limits of the noisy-storage model using entanglement sampling. In Advances in Cryptology - CRYPTO 2013, volume 8043 of LNCS, pages 326-343. Springer, 2013. DOI : $10.1007 / 978-3-642-40084-1 \_19$.

[15] U. Feige and M. Seltser. On the densest k-subgraph problem. Algorithmica, 29:2001, 1997.

[16] D. Gavinsky, J. Kempe, I. Kerenidis, R. Raz, and R. de Wolf. Exponential separations for one-way quantum communication complexity, with applications to cryptography. In Proceedings of the thirty-ninth annual ACM symposium on Theory of computing, STOC '07, pages 516-525. ACM, 2007. DOI: 10.1145/1250790.1250866.

[17] M. Junge. Operator spaces and Araki-Woods factors: a quantum probabilistic approach. IMRP. International Mathematics Research Papers, pages Art. ID 76978-87, 2006.

[18] M. Junge and C. Palazuelos. Large violation of Bell inequalities with low entanglement. Communications in Mathematical Physics, 306:695-746, 2011. DOI : 10.1007/s00220-011-1296-8.

[19] M. Junge, C. Palazuelos, D. Pérez-García, I. Villanueva, and M. M. Wolf. Unbounded violations of bipartite Bell inequalities via operator space theory. Communications in Mathematical Physics, 300:715-739, 2010. DOI : $10.1007 / \mathrm{s} 00220-010-1125-5$.

[20] M. Junge and J. Parcet. Mixed-norm inequalities and operator space $\mathrm{L}_{p}$ embedding theory. Memoirs of the American Mathematical Society, 203:vi-155, 2010.

[21] A. Kitaev. Quantum computations: algorithms and error correction. Russian Mathematical Surveys, 52(6):1191, 1997. Available online: http://stacks.iop.org/0036-0279/52/i=6/a=R02.

[22] R. Konig and R. Renner. Sampling of min-entropy relative to quantum knowledge. Information Theory, IEEE Transactions on, 57:4760-4787, 2011. DOI: 10.1109/TIT.2011.2146730.

[23] R. Konig and B. Terhal. The bounded-storage model in the presence of a quantum adversary. Information Theory, IEEE Transactions on, 54:749-762, 2008. DOI : 10.1109/TIT.2007.913245.

[24] R. Konig, S. Wehner, and J. Wullschleger. Unconditional security from noisy quantum storage. Information Theory, IEEE Transactions on, 58:1962-1984, 2012. DOI : 10.1109/TIT.2011.2177772.

[25] C. A. Miller and Y. Shi. Robust protocols for securely expanding randomness and distributing keys using untrusted quantum devices. 2014. Available online: http://arxiv.org/abs/1402.0489.

[26] N. Nisan and A. Ta-Shma. Extracting randomness: a survey and new constructions. Journal of Computer and System Sciences, 58(1, part 2):148-173, 1999.

[27] N. Nisan and D. Zuckerman. Randomness is linear in space. Journal of Computer and System Sciences, 51:43 - 52, 1996. DOI : $10.1006 /$ jcss.1996.0004.

[28] G. Pisier. Introduction to operator space theory, volume 294. Cambridge University Press, 2003.

[29] G. Pisier. Grothendieck's theorem, past and present. Bulletin of the American Mathematical Society, 49:237-323, 2012. DOI : $10.1090 /$ S0273-0979-2011-01348-9.

[30] J. Radhakrishnan and A. Ta-Shma. Bounds for dispersers, extractors, and depth-two superconcentrators. SIAM Journal on Discrete Mathematics, 13:2-24, 2000. DOI : 10.1137/S0895480197329508.

[31] R. Raz and O. Reingold. On recycling the randomness of states in space bounded computation. In Proceedings of the thirty-first annual ACM symposium on Theory of computing, pages 159-168. ACM, 1999. DOI: 10.1145/301250.301294.

[32] O. Reingold, R. Shaltiel, and A. Wigderson. Extracting randomness via repeated condensing. SIAM Journal on Computing, 35:1185-1209, 2006. DOI : 10.1137/S0097539703431032.

[33] R. Renner. Security of Quantum Key Distribution. PhD thesis, ETH Zurich, 2005. D0I: 10.1142/S0219749908003256.

[34] Z.-J. Ruan. Subspaces of C*-algebras. Journal of functional analysis, 76:217-230, 1988.

[35] R. Shaltiel. Recent developments in explicit constructions of extractors. Bulletin of the EATCS, 77:67-95, 2002. Available 
online: http://kam.mff.cuni.cz/ matousek/cla/shaltiel-extractors-survey.ps.

[36] M. Sipser. Expanders, randomness, or time versus space. Journal of Computer and System Sciences, $36: 379$ - $383,1988$. DOI : $10.1016 / 0022-0000$ (88) 90035-9.

[37] O. Szehr. Decoupling theorems. Master's thesis, ETH Zurich, 2011. Available online: http://arxiv.org/abs/1207.3927.

[38] A. Ta-Shma. Extractors against classical and quantum adversaries. Tutorial QCrypt, 2013. Available online: http://2013.qcrypt.net/contributions/Ta-Shma-slides.pptx.

[39] A. Ta-Shma, C. Umans, and D. Zuckerman. Lossless condensers, unbalanced expanders, and extractors. Combinatorica, 27:213-240, 2007.

[40] M. Tomamichel, C. Schaffner, A. Smith, and R. Renner. Leftover hashing against quantum side information. Information Theory, IEEE Transactions on, 57:5524 -5535, 2011. DOI: 10.1109/TIT.2011.2158473.

[41] L. Trevisan. Construction of extractors using pseudo-random generators (extended abstract). In Proceedings of the thirty-first annual ACM symposium on Theory of computing, STOC '99, pages 141-148. ACM, 1999. DOI : $10.1145 / 301250.301289$.

[42] S. Vadhan. The unified theory of pseudorandomness: guest column. SIGACT News, 38:39-54, 2007. DOI : $10.1145 / 1324215.1324225$.

[43] U. Vazirani and T. Vidick. Certifiable quantum dice: Or, true random number generation secure against quantum adversaries. In Proceedings of the Forty-fourth Annual ACM Symposium on Theory of Computing, STOC '12, pages 61-76, New York, NY, USA, 2012. ACM. DOI : 10.1145/2213977.2213984.

[44] J. Watrous. Notes on super-operator norms induced by schatten norms. Quantum Information E5 Computation, 5:58-68, 2005.

[45] J. Watrous. Semidefinite programs for completely bounded norms. Theory of Computing, 5:217-238, 2009. DOI : $10.4086 /$ toc. 2009.v005a011.

[46] J. Wullschleger. Bitwise quantum min-entropy sampling and new lower bounds for random access codes. In 6 th Conference, TQC 2011, Revised Selected Papers. Springer, 2014. DOI : 10.1007/978-3-642-54429-3_11. 